\title{
On the Edgeworth Expansion and the $M$ out of $N$ Bootstrap Accuracy for a Studentized Trimmed Mean
}

\author{
N. V. Gribkova ${ }^{1 *}$ and R. Helmers ${ }^{2 * *}$ \\ ${ }^{1}$ St.Petersburg State Transport University, \\ 190031, Moskovskii av. 9, St.Petersburg, Russia \\ ${ }^{2}$ Centre for Mathematics and Computer Science, \\ P.O.Box 94079, 1090 GB Amsterdam, The Netherlands \\ Received December 21, 2006
}

\begin{abstract}
We investigate the second order accuracy of the $M$ out of $N$ bootstrap for a Studentized trimmed mean using the Edgeworth expansion derived in a previous paper. Some simulations, which support our theoretical results, are also given. The effect of extrapolation in conjunction with the $M$ out of $N$ bootstrap for Studentized trimmed means is briefly discussed. As an auxiliary result we obtain a Bahadur's type representation for an $M$ out of $N$ bootstrap quantile.

Our results supplement previous work on (Studentized) trimmed means by Hall and Padmanabhan [13], Bickel and Sakov [7], and Gribkova and Helmers [11].

Key words: bootstrap accuracy, empirical Edgeworth expansions, Studentized trimmed means. 2000 Mathematics Subject Classification: primary 62E20, 62G30, 62G09; secondary 60F05.
\end{abstract}

DOI: $10.3103 / \mathrm{S} 1066530707020056$

\section{INTRODUCTION}

This article is closely connected with our previous paper [11], where the validity of the one-term Edgeworth expansion (EE) and the empirical Edgeworth expansion (EEE) for a Studentized trimmed mean was established and explicit formulas for the leading terms of the EE were obtained. We study two second-order approximations to the distribution function $(d f)$ of a Studentized trimmed mean: EE and bootstrap.

During the past twenty five years the attention of many authors was focussed on Efron's bootstrap ([8]), and nowadays there exists a voluminous literature on this topic. The consistency of the standard nonparametric, or naive, bootstrap was proved for many interesting statistics, at least for the asymptotically normal ones (see $[1,4,6,7,12,13,15,25]$ and references therein). One of the main reasons of interest in the bootstrap and its application in statistics is the second order accuracy property: under proper conditions the bootstrap approximation to the distribution function $(d f)$ of a pivotal statistic is more accurate than the normal one. This beneficial property of the bootstrap was proved for the sample mean [25], for the class of Hoeffding's $U$-statistics (cf. [15]) and for some other statistics. The usual way to prove this fact is based on the congruence of the one-term EE for the pivotal statistic (in the 'real world') and the EE for its bootstrap counterpart (in the 'bootstrap world'). Typically the structure of the one-term EE for the bootstrapped statistic is the same as the one for the pivotal statistic, when the parameters of the formula of the first leading term of EE are replaced by their empirical counterparts (plug-in estimators). So, the application of a relevant version of the Law of Large Numbers implies the second order accuracy of the bootstrap (cf. $[12,15,25])$. However, the case of the trimmed mean is a special one. The problem is connected with the difficulty in obtaining the explicit formula for the oneterm EE (cf. [13]).

In this paper we establish the validity of a one-term EE for the bootstrapped Studentized trimmed mean (in the bootstrap world). We also obtain an explicit formula for the $M^{-1 / 2}$-term (correcting for

\footnotetext{
${ }^{*}$ E-mail: nvg@rol.ru

${ }^{* * *}$ E-mail: helmers@cwi.nl
} 
skewness and bias; $M$ being the size of the bootstrap sample). Using this expansion and our previous results [11] (extended and slightly improved here) we prove that $M$ out of $N$ bootstrap approximation to the $d f$ of the trimmed mean and Studentized trimmed mean is more accurate than the normal one, provided a natural condition (cf. $(C .2)$ ) on the size of the bootstrap resample $M$ in relation to size $N$ is satisfied. Our results can be viewed as a mathematical contribution to the asymptotic theory for the $M$ out of $N$ bootstrap. In a way the only thing we do is proving for Studentized trimmed mean under minimal conditions what statisticians expect to be true in nice asymptotically normal situations.

The second order accuracy of the bootstrap for a Studentized trimmed mean was also established by Hall and Padmanabhan [13] for the special case of the naive bootstrap, i.e., when $M=N$. Their proof relies on the existence of the EE for a Studentized trimmed mean.

Our main results are more general and precise than those in [13]. In Theorem 2.5 we assume that $M=N+O\left(N^{r}\right)$ for some $r<1$ and that the density of the underlying distribution exists and is positive and Hölder continuous near the two quantiles, where trimming occurs. Moreover, we establish the order of magnitude of the $M$ out of $N$ bootstrap error in terms of $r$ and $a$ (the parameter appearing in the Hölder condition). On the other hand, in Theorems 2.7 and 2.8, we focus on the case that $M$ is of smaller order of magnitude than $N$. We establish the asymptotic accuracy for the $M$ out of $N$ bootstrap in conjunction with extrapolation, and we show that it will lead to a better performance. Our results are completed with simulations.

The paper is organized as follows: in Section 2, we state our main results on the EE and $M$ out of $N$ bootstrap, and we discuss the important special case of the naive bootstrap. In Section 3 , we present some numerical results based on simulations. In Section 4, we state and prove Bahadur-type lemmas. A lemma on bias approximation is also presented in Section 4. In Section 5, a $U$-statistic type approximation to the bootstrapped trimmed mean is established, and our result for a normalized version of bootstrapped trimmed mean is proved. Those parts of the proof which are similar to the corresponding ones in [11] are discussed only briefly. In Section 6, a stochastic approximation for a plug-in estimator, which is used to construct a Studentized bootstrapped trimmed mean is established, and the result on the EE for a Studentized bootstrapped version of the statistic is proved. In Section 7, the bootstrap versions of the Bahadur-type lemmas are proved. In Appendix, we state and prove a lemma on conditioning.

\section{MAIN RESULTS}

Let $X_{1}, X_{2}, \ldots$ denote a sequence of independent and identically distributed (i.i.d.) realvalued random variables (r.v.) with common distribution function $(d f) F$, and let $X_{1: N} \leq \cdots \leq X_{N: N}$ $(N=1,2, \ldots)$ be the corresponding order statistics. Let $X_{1}^{*}, \ldots, X_{M}^{*}$ be a bootstrap resample of size $M=M(N)$ from the empirical $d f F_{N}$ based on the first $N$ original observations $X_{1}, \ldots, X_{N}$; denote by $F_{M}^{*}$ the bootstrap empirical $d f$, i.e., $F_{M}^{*}(x)=M^{-1} \sharp\left\{i: X_{i}^{*} \leq x, 1 \leq i \leq M\right\},-\infty<x<\infty$, and let $X_{1: M}^{*} \leq \cdots \leq X_{M: M}^{*}$ be the corresponding order statistics. Here and throughout this paper we use the shorthand notation $M$, omitting its argument $N$. The $M$ out of $N$ bootstrap (with replacement) will be shown to be second-order accurate (cf. Theorems 2.5 and 2.7), like the classical naive bootstrap $M=N$, with reduced computation time (cf., for instance, Bickel and Sakov [7]).

Let $F^{-1}(u)=\inf \{x: F(x) \geq u\}, 0<u \leq 1$, denote the left-continuous inverse function of the $d f F$ and put $F_{N}^{-1},\left(F_{M}^{*}\right)^{-1}$ to be the inverse functions of $F_{N}$ and $F_{M}^{*}$, respectively.

Consider the trimmed mean given by

$$
T_{N}=\frac{1}{\beta-\alpha} \int_{\alpha}^{\beta} F_{N}^{-1}(u) d u
$$

which is precisely equal to

$$
-c_{\alpha, N} X_{[\alpha N]+1: N}+\frac{1}{(\beta-\alpha) N} \sum_{i=[\alpha N]+1}^{[\beta N]} X_{i: N}+c_{\beta, N} X_{[\beta N]+1: N},
$$


where $0<\alpha<\beta<1$ are any fixed numbers, $[\cdot]$ represents the greatest integer function and $c_{\nu, N}=$ $(\nu N-[\nu N]) /((\beta-\alpha) N), \quad \nu=\alpha, \beta$. This version of the trimmed mean also occurs in $[18,14]$ (cf. also [4]). Indeed, (2.1) is a natural definition for the trimmed mean in a bootstrap context.

In [11] we studied the Edgeworth expansion (EE) for the trimmed mean given by

$$
S_{N}=([\beta N]-[\alpha N])^{-1} \sum_{i=[\alpha N]+1}^{[\beta N]} X_{i: N},
$$

which is a more common way of defining a trimmed mean (cf. also [26, 13, 23]). The reason why we now prefer $T_{N}$ (cf. (2.1)) is its convenience for the bootstrap. This will be discussed in the paragraph after Theorem 2.5, our first main result on the $M$ out of $N$ bootstrap.

It is well known that a trimmed mean given by (2.1) (or the one defined as $S_{N}$ ) often serves as a statistical estimator of the location parameter

$$
\mu(\alpha, \beta)=\frac{1}{\beta-\alpha} \int_{\alpha}^{\beta} F^{-1}(u) d u .
$$

Let us introduce the $\nu$-th $(0<\nu<1)$ quantile of $F$ by $\xi_{\nu}=F^{-1}(\nu)$, and define the sample quantile $\xi_{\nu N: N}=F_{N}^{-1}(\nu)$ and the bootstrap quantile $\xi_{\nu M: M}^{*}=\left(F_{M}^{*}\right)^{-1}(\nu)$.

The $M$ out of $N$ bootstrap counterpart of $T_{N}$ is given by

$$
T_{N, M}^{*}=\frac{1}{\beta-\alpha} \int_{\alpha}^{\beta}\left(F_{M}^{*}\right)^{-1}(u) d u,
$$

which is equal to

$$
-c_{\alpha, M} X_{[\alpha M]+1: M}^{*}+\frac{1}{(\beta-\alpha) M} \sum_{i=[\alpha M]+1}^{[\beta M]} X_{i: M}^{*}+c_{\beta, M} X_{[\beta M]+1: M}^{*},
$$

with $c_{\nu, M}=(\nu M-[\nu M]) /((\beta-\alpha) M), \nu=\alpha, \beta$.

Suppose that $\xi_{\alpha} \neq \xi_{\beta}$ (that is $\xi_{\alpha}$ is not an atom of the distribution $F$ with mass at least $(\beta-\alpha)$ ), and let $W_{i}$ and $W_{i}^{*}$ be $X_{i}$ and $X_{i}^{*}$ Winsorized outside of $\left(\xi_{\alpha}, \xi_{\beta}\right]$ and $\left(\xi_{\alpha N: N}, \xi_{\beta N: N}\right]$ respectively. In other words

$$
\begin{aligned}
W_{i} & =\xi_{\alpha} \vee\left(X_{i} \wedge \xi_{\beta}\right), \quad i=1, \ldots, N, \\
W_{i}^{*} & =\xi_{\alpha N: N} \vee\left(X_{i}^{*} \wedge \xi_{\beta N: N}\right), \quad i=1, \ldots, M,
\end{aligned}
$$

where $a \wedge b=\min (a, b)$ and $a \vee b=\max (a, b)$.

Finally, define the quantile function $Q(u)$, the empirical quantile function $Q_{N}(u)$, and its bootstrap version $Q_{M}^{*}(u)$ by

$$
\begin{gathered}
Q(u)=\xi_{\alpha} \vee\left(F^{-1}(u) \wedge \xi_{\beta}\right), \quad Q_{N}(u)=\xi_{\alpha N: N} \vee\left(F_{N}^{-1}(u) \wedge \xi_{\beta N: N}\right), \\
Q_{M}^{*}(u)=\xi_{\alpha M: M}^{*} \vee\left(\left(F_{M}^{*}\right)^{-1}(u) \wedge \xi_{\beta M: M}^{*}\right),
\end{gathered}
$$

for $0<u \leq 1$. The first three central moments of $W_{1}$ are given by

$$
\mu_{W}=\int_{0}^{1} Q(u) d u, \quad \sigma_{W}^{2}=\int_{0}^{1}\left(Q(u)-\mu_{W}\right)^{2} d u, \quad \gamma_{W}=\int_{0}^{1}\left(Q(u)-\mu_{W}\right)^{3} d u
$$

and the corresponding moments of $W_{1}^{*}$ are equal to

$$
\mu_{W, N}=\int_{0}^{1} Q_{N}(u) d u, \quad \sigma_{W, N}^{2}=\int_{0}^{1}\left(Q_{N}(u)-\mu_{W, N}\right)^{2} d u, \quad \gamma_{W, N}=\int_{0}^{1}\left(Q_{N}(u)-\mu_{W, N}\right)^{3} d u
$$


Define the $d f$ 's of the normalized trimmed mean and its bootstrap counterpart by

$$
F_{T_{N}}(x)=P\left(\frac{N^{1 / 2}\left(T_{N}-\mu(\alpha, \beta)\right)}{(\beta-\alpha)^{-1} \sigma_{W}} \leq x\right) \quad \text { and } \quad F_{T_{N, M}^{*}}^{*}(x)=P^{*}\left(\frac{M^{1 / 2}\left(T_{N, M}^{*}-T_{N}\right)}{(\beta-\alpha)^{-1} \sigma_{W, N}} \leq x\right) .
$$

Here and elsewhere $P^{*}$ denotes the bootstrap probability measure having discrete mass points $X_{i: N}$ with atoms $1 / N$, and $E^{*}$ denotes the corresponding expectation.

To establish our results on the EE we will assume that the following smoothness condition is satisfied:

(C.1) Suppose that the distribution function $F$ has a density $f=F^{\prime}$ in some neighborhoods of the points $\xi_{\nu}$, with $f\left(\xi_{\nu}\right)>0, \nu=\alpha, \beta$. In addition, we assume that the density $f$ satisfies Hölder's condition of order $a>0$ at the points $\xi_{\alpha}, \xi_{\beta}$, i.e., there exists a constant $C$ such that $\left|f(x)-f\left(\xi_{\nu}\right)\right| \leq C\left|x-\xi_{\nu}\right|^{a}$, for all $x$ in a neighborhood of $\xi_{\nu}, \nu=\alpha, \beta$.

For the validity of our results on the bootstrap accuracy we will need a slightly stronger smoothness condition:

$\left(C .1^{\prime}\right)$ In addition to (C.1) assume that the density $f$ satisfies a uniform Hölder condition of order $a>0$ in neighborhoods of the points $\xi_{\alpha}$, $\xi_{\beta}$, i.e., there exists a constant $C$ such that $|f(x)-f(y)| \leq C|x-y|^{a}$, for all $x, y$ in a neighborhood of $\xi_{\nu}, \nu=\alpha, \beta$.

Moreover, the following condition on the growth rate of $M$ as a function of $N$ will be required:

(C.2) For some $0<d \leq 1$

$$
M=O\left(N^{2-d}\right)
$$

as $\min (N, M) \rightarrow \infty$.

The purpose of condition (C.2) is to ensure that an appropriate version (cf. Lemma 4.2) of Bahadur's representation for $M$ out of $N$ bootstrap quantile is valid. If $(C .2)$ does not hold - for instance when $M=N^{2}$ - the remainder term of our expansions will be of the same order, viz. $1 / N$ in case $M=N^{2}$, as the first term in the expansions. Note that $(C .2)$ implies $1 / N=o\left(M^{-1 / 2-p}\right)$ for every $p<d /(2(2-d))$. Technically speaking $(C .2)$ enables us to obtain an error term for our expansions of order $M^{-1 / 2-p}$ in the bootstrap world (cf. also the remark following Lemma 7.1).

To state our results on the Edgeworth expansions and their bootstrap versions we need the following notations. Set

$$
\begin{gathered}
\delta_{W}=-\alpha^{2} \frac{1}{f\left(\xi_{\alpha}\right)}\left[\mu_{W}-\xi_{\alpha}\right]^{2}+(1-\beta)^{2} \frac{1}{f\left(\xi_{\beta}\right)}\left[\mu_{W}-\xi_{\beta}\right]^{2}, \\
\delta_{W, N}=-\alpha_{N}^{2} \frac{1}{f\left(\xi_{\alpha N: N)}\right)}\left[\mu_{W, N}-\xi_{\alpha N: N}\right]^{2}+\left(1-\beta_{N}\right)^{2} \frac{1}{f\left(\xi_{\beta N: N}\right)}\left[\mu_{W, N}-\xi_{\beta N: N}\right]^{2},
\end{gathered}
$$

where $\nu_{N}=F_{N}\left(\xi_{\nu N: N}\right), \nu=\alpha, \beta$. Define real numbers $\lambda_{1}$ and $\lambda_{2}$ by

$$
\lambda_{1}=\gamma_{W} / \sigma_{W}^{3}, \quad \lambda_{2}=\delta_{W} / \sigma_{W}^{3}
$$

(cf. $[11,21])$ and their empirical counterparts by

$$
\lambda_{1, N}=\gamma_{W, N} / \sigma_{W, N}^{3}, \quad \lambda_{2, N}=\delta_{W, N} / \sigma_{W, N}^{3} .
$$

Note that, in contrast to (2.7), (2.11) is not completely empirical, as it involves the values of the density $f$ at the two quantiles, where the trimming occurs; one way of estimating these unknown values is discussed in [11]. It was established in Gribkova and Helmers [11] that the Edgeworth expansion for the df $F_{T_{N}}(x)$ is given by

$$
G_{N}(x)=\Phi(x)-\frac{\phi(x)}{6 \sqrt{N}}\left(\left(\lambda_{1}+3 \lambda_{2}\right)\left(x^{2}-1\right)+6 \sqrt{N} \frac{b_{N}}{\sigma_{W}}\right),
$$


where $\Phi$ is the standard normal distribution function, $\phi=\Phi^{\prime}$ its density and $b_{N}$ is a bias term given by

$$
b_{N}=\frac{1}{2 \sqrt{N}}\left\{-\alpha(1-\alpha) \frac{1}{f\left(\xi_{\alpha}\right)}+\beta(1-\beta) \frac{1}{f\left(\xi_{\beta}\right)}\right\} .
$$

To be more precise, in [11] the validity of the one-term EE and the explicit formula $G_{N}(x)$ are established for the trimmed mean $S_{N}$ defined by

$$
S_{N}=\frac{1}{([\beta N]-[\alpha N])} \sum_{i=[\alpha N]+1}^{[\beta N]} X_{i: N} .
$$

However, it is clear from our proofs that the one-term EE's for $T_{N}$ and $S_{N}$ are the same in their skewness terms, and differ only in the bias terms. A similar comment applies to the Studentized $T_{N}$ and $S_{N}$ and their bootstrap counterparts. It was shown in [11] that the bias of $S_{N}$ in estimating of $\mu(\alpha, \beta)$ is given by

$$
d_{N}=b_{N}+b_{[\cdot], N}
$$

with $b_{N}$ as in (2.13) and

$$
b_{[\cdot], N}=\frac{1}{\sqrt{N}}\left\{-(\alpha N-[\alpha N])\left(\mu(\alpha, \beta)-\xi_{\alpha}\right)+(\beta N-[\beta N])\left(\mu(\alpha, \beta)-\xi_{\beta}\right)\right\}
$$

(cf. Lemma A.1, [11], see also Lemma 4.5, Section 4).

The following result is a refined version of Theorem 2.1 from [11] on the Edgeworth expansion for the normalized trimmed mean.

Theorem 2.1. Suppose that condition (C.1) holds true. Then

$$
\sup _{x \in R}\left|F_{T_{N}}(x)-G_{N}(x)\right|=o\left(N^{-1 / 2-p}\right)
$$

for every $p<\min (a / 2,1 / 4)$ as $N \rightarrow \infty$.

Let us compare this result with Theorem 2.1 in [11], where the one-term Edgeworth expansion for the normalized trimmed mean was obtained. In [11] we assumed that the underlying distribution has a density, which is positive and Lipschitz in neighborhoods of $\xi_{\alpha}$ and $\xi_{\beta}$. Under this condition we obtained that the remainder term of the EE is of the classical Bahadur's order $O\left((\log N)^{5 / 4} N^{-3 / 4}\right)$. In the present paper our smoothness condition is slightly weaker: we suppose that the density is Hölder continuous of degree $a>0$ at the points $\xi_{\alpha}$ and $\xi_{\beta}$ and establish the order of magnitude of the remainder term in terms of the parameter $a$ appearing in the Hölder condition ( $\mathrm{cf}$. (C.1)). At the same time we find that the classical Bahadur order for the remainder term holds true whenever $a \geq 1 / 2$, while in [11] the same fact was obtained for the special case $a=1$.

To prove Theorem 2.1 it suffices to repeat the arguments used for proving Theorem 2.1 in [11], replacing the application of Lemmas 3.1, 3.2, and A.1 from [11] by applying Lemmas 4.1, 4.3, and 4.5, respectively, of the present paper (see Section 4).

Remark 2.1. It is clear from our proofs (cf. also the proof of Theorem 2.1 in [11]) that the r.h.s. in (2.16) is in fact of order

$$
O\left(\frac{\log N}{N^{1 / 2}}\left((\log N / N)^{1 / 4}+(\log N / N)^{a / 2}\right)\right),
$$

i.e., we obtain a slightly stronger result than the one stated in Theorem 2.1. A similar comment applies to our results for the 'bootstrap world'.

Let us now consider the $M$ out of $N$ bootstrapped trimmed mean given by (2.3). The classical result of Stigler [26] directly implies that if the inverse function $F^{-1}$ is continuous at the two points $\alpha$ and $\beta$ and $\min (N, M)$ tends to infinity, the limiting distribution of the normalized (and also of a Studentized) $T_{N, M}^{*}$ is standard normal with probability one. This fact yields the first order accuracy of the bootstrap 
approximation for the $d f$ of the trimmed mean. In the present paper we prove that if conditions $\left(C .1^{\prime}\right)$ and $(C .2)$ hold true, then the Edgeworth expansion for the $d f$ of a normalized version of the bootstrapped trimmed mean is given by

$$
G_{N, M}(x)=\Phi(x)-\frac{\phi(x)}{6 \sqrt{M}}\left(\left(\lambda_{1, N}+3 \lambda_{2, N}\right)\left(x^{2}-1\right)+6 \sqrt{M} \frac{b_{N, M}}{\sigma_{W, N}}\right),
$$

where $b_{N, M}$ is the bias term given by

$$
b_{N, M}=\frac{1}{2 \sqrt{M}}\left\{-\alpha_{N}\left(1-\alpha_{N}\right) \frac{1}{f\left(\xi_{\alpha N: N}\right)}+\beta_{N}\left(1-\beta_{N}\right) \frac{1}{f\left(\xi_{\beta N: N}\right)}\right\} .
$$

In Lemma 4.5 we show that if $\left(C .1^{\prime}\right)$ and $(C .2)$ hold, then

$$
M^{1 / 2}(\beta-\alpha)\left(E^{*} T_{N, M}^{*}-T_{N}\right)-b_{N, M}=o\left(M^{-1 / 2-p}\right)
$$

for every

$$
p<\min \left(\frac{a}{2}, \frac{1}{4}, \frac{d}{4(2-d)}\right) \quad \text { a.s. }[P],
$$

as $\min (N, M) \rightarrow \infty$, where $a>0$ is the parameter in $\left(C .1^{\prime}\right)$, and $d$ is the parameter in $(C .2)$. Inequality (2.20) describes the dependence of the order of magnitude of the error in the bootstrap bias estimate on the parameters $a$ and $d$.

The next assertion is a bootstrap version of Theorem 2.1.

Theorem 2.2. Suppose that conditions $\left(C .1^{\prime}\right)$ and $(C .2)$ hold true. Then for every $p$ satisfying $(2.20)$

$$
\sup _{x \in R}\left|F_{T_{N, M}^{*}}^{*}(x)-G_{N, M}(x)\right|=o\left(M^{-1 / 2-p}\right)
$$

with probability one, as $\min (N, M) \rightarrow \infty$.

Note that if $d=1$ (i.e., $M=O(N)$ as $\min (N, M) \rightarrow \infty$ ) and $a \geq 1 / 2$, then

$$
\min \left(\frac{1}{4}, \frac{d}{4(2-d)}\right)=\frac{1}{4}
$$

(cf. (2.20)), therefore relation (2.21) gives a bound of Bahadur's order in $M$. The proof of Theorem 2.2 can be found in Section 5 .

Note also that in fact we prove in Section 5 a slightly stronger assertion, namely, that for every $c>0$ and $p$ satisfying $(2.20)$

$$
P\left(\sup _{x \in R}\left|F_{T_{N, M}^{*}}^{*}(x)-G_{N, M}(x)\right|>M^{-1 / 2-p}\right)=o\left(N^{-c}\right)
$$

as $\min (N, M) \rightarrow \infty$, which by the Borel-Cantelli lemma, with $c>1$, directly yields (2.21). A similar comment applies to other our results for the bootstrap world.

Now we state our results for a Studentized trimmed mean and the bootstrapped Studentized trimmed mean. Define the $d f$ of the Studentized $T_{N}$ by

$$
F_{T_{N}, S}(x)=P\left(\frac{N^{1 / 2}\left(T_{N}-\mu(\alpha, \beta)\right)}{(\beta-\alpha)^{-1} \sigma_{W, N}} \leq x\right)
$$

and the $d f$ of the Studentized $T_{N, M}^{*}$ by

$$
F_{T_{N, M}^{*}, S}^{*}(x)=P^{*}\left(\frac{M^{1 / 2}\left(T_{N, M}^{*}-T_{N}\right)}{(\beta-\alpha)^{-1} \sigma_{W, M}^{*}} \leq x\right)
$$


respectively, where

$$
\left(\sigma_{W, M}^{*}\right)^{2}=\int_{0}^{1}\left(Q_{M}^{*}(u)-\mu_{W, M}^{*}\right)^{2} d u \quad \text { and } \quad \mu_{W, M}^{*}=\int_{0}^{1} Q_{M}^{*}(u) d u
$$

with $Q_{M}^{*}$ as in (2.5). It was shown in [11] that the Edgeworth expansion for a Studentized trimmed mean is given by

$$
H_{N}(x)=\Phi(x)+\frac{\phi(x)}{6 \sqrt{N}}\left(\left(2 x^{2}+1\right) \lambda_{1}+3\left(x^{2}+1\right) \lambda_{2}-6 \sqrt{N} \frac{b_{N}}{\sigma_{W}}\right) .
$$

The following result is a refined version of Theorem 2.2 in [11].

Theorem 2.3. Suppose that condition (C.1) holds true. Then

$$
\sup _{x \in R}\left|F_{T_{N}, S}(x)-H_{N}(x)\right|=o\left(N^{-1 / 2-p}\right)
$$

for every $p<\min (a / 2,1 / 4)$ as $N \rightarrow \infty$.

To prove relation (2.23), it suffices to repeat the argument used when proving Theorem 2.2 in [11], applying now Lemmas 4.1, 4.3, and 4.5 (cf. Section 4) instead of Lemmas 3.1, 3.2, and A.1 (cf. [11]).

The Edgeworth expansion for the bootstrapped Studentized trimmed mean is given by

$$
H_{N, M}(x)=\Phi(x)+\frac{\phi(x)}{6 \sqrt{M}}\left(\left(2 x^{2}+1\right) \lambda_{1, N}+3\left(x^{2}+1\right) \lambda_{2, N}-6 \sqrt{M} \frac{b_{N, M}}{\sigma_{W, N}}\right) .
$$

The next assertion is a bootstrap version of Theorem 2.3.

Theorem 2.4. Suppose that conditions $\left(C .1^{\prime}\right)$ and (C.2) hold true. Then for every $p$ satisfying $(2.20)$

$$
\sup _{x \in R}\left|F_{T_{N, M}^{*}, S}^{*}(x)-H_{N, M}(x)\right|=o\left(M^{-1 / 2-p}\right)
$$

with probability one as $\min (N, M) \rightarrow \infty$.

The proof of Theorem 2.4 can be found in Section 6 .

Note that if $(C .1)$ holds with $a \geq 1 / 2$, the order of our approximation for the normalized and Studentized trimmed mean is $o\left(N^{-3 / 4+\varepsilon}\right)$ for every $\varepsilon>0$ (i.e., is of the classical Bahadur's [2] order), and if in addition $d=1$ (that is $M=O(N)$ whenever $\min (N, M) \rightarrow \infty$ ), our approximation for the $d f$ of the bootstrap version of the trimmed mean is of order $o\left(M^{-3 / 4+\varepsilon}\right)$ for every $\varepsilon>0$.

Theorem 2.4 can be compared with the main result of Hall and Padmanabhan [13], where the existence of the asymptotic expansion for the $d f$ of a Studentized trimmed mean and its bootstrap version was established. They restrict attention to the case $M=N$ and obtain a bootstrap approximation with the error of order $o\left(N^{-1 / 2-\varepsilon}\right)$ (for some $\varepsilon>0$ ) under $\left(C \cdot 1^{\prime}\right)$ with $a=1$.

Remark 2.2. It is easy to verify from the proofs of Theorems 2.2 and 2.4 that the relations (2.21) and (2.25) are valid also for the $d f$ 's of the bootstrap versions of the trimmed mean given by

$$
S_{N, M}^{*}=([\beta M]-[\alpha M])^{-1} \sum_{i=[\alpha M]+1}^{[\beta M]} X_{i: M}^{*},
$$

provided we replace $b_{N, M}$ (the bias term) in formulas (2.18) and (2.24) by

$$
d_{N, M}=b_{N, M}+b_{[\cdot], N, M},
$$

with

$$
b_{[\cdot], N, M}=\frac{1}{\sqrt{M}}\left\{-(\alpha M-[\alpha M])\left(T_{N}-\xi_{\alpha N: N}\right)+(\beta M-[\beta M])\left(T_{N}-\xi_{\beta N: N}\right)\right\},
$$

where $T_{N}$ is defined by (2.1) and $b_{N, M}$ as in (2.19) (cf. (2.15)). 
Define the quantity

$$
b=\frac{\sqrt{N} b_{N}}{\sigma_{W}}=\frac{1}{2 \sigma_{W}}\left(-\frac{\alpha(1-\alpha)}{f\left(\xi_{\alpha}\right)}+\frac{\beta(1-\beta)}{f\left(\xi_{\beta}\right)}\right) .
$$

The following corollary is a simple consequence of our previous results.

Corollary 2.1. Suppose that conditions $\left(C .1^{\prime}\right)$ and $(C .2)$ hold true. Then

$$
\begin{aligned}
F_{T_{N, M}^{*}}^{*}(x)-F_{T_{N}}(x)=- & \phi(x)\left(\frac{1}{\sqrt{M}}-\frac{1}{\sqrt{N}}\right)\left[\frac{1}{6}\left(\lambda_{1}+3 \lambda_{2}\right)\left(x^{2}-1\right)+b\right] \\
+ & R_{1, N}+R_{1, N, M}+R_{1, M}, \\
F_{T_{N, M}^{*}, S}^{*}(x)-F_{T_{N}, S}(x)=\phi( & x)\left(\frac{1}{\sqrt{M}}-\frac{1}{\sqrt{N}}\right)\left[\frac{1}{6} \lambda_{1}\left(2 x^{2}+1\right)+\frac{1}{2} \lambda_{2}\left(x^{2}+1\right)-b\right] \\
+ & R_{2, N}+R_{2, N, M}+R_{2, M},
\end{aligned}
$$

where, for $j=1,2, R_{j, N}=o\left(N^{-1 / 2-p}\right)$ for every $p<\min (a / 2,1 / 4)$ as $N \rightarrow \infty$, and $R_{j, N, M}=$ $o\left(M^{-1 / 2} N^{-s}\right)$ for every $s<a / 2$, a.s. $[P]$, as $\min (N, M) \rightarrow \infty, R_{j, M}=o\left(M^{-1 / 2-p}\right)$ for every $p$ satisfying (2.20), a.s. $[P]$, as $\min (N, M) \rightarrow \infty$.

Note that $M$ can be of smaller order than $N$ (cf. Theorem 2.7), as well as of larger order, i.e., $M \gg N$. The border case $M=N$ corresponds to the standard nonparametric naive Efron's bootstrap resampling plan. Corollary 2.1 follows directly from Theorems $2.1-2.4$, our smoothness condition, and Lemma 6.2 in [11], where in the proof of this lemma (cf. [11]) we now apply Lemmas 4.1 and 4.3 of the present paper instead of the related lemmas from [11], because in [11] we require the stronger assumption that $f$ is Lipschitz in neighborhoods of $\xi_{\alpha}, \xi_{\beta}$, while here we assume only that it is Hölder continuous.

Corollary 2.1 directly implies the following consequence.

Corollary 2.2. Suppose that conditions $\left(C .1^{\prime}\right)$ and (C.2) hold true. If $\frac{2 b}{\lambda_{1}+3 \lambda_{2}} \geq 1$ or $\lambda_{1}+3 \lambda_{2}=0$, then

$$
\sup _{x \in R}\left|F_{T_{N, M}^{*}}^{*}(x)-F_{T_{N}}(x)\right|=\frac{1}{\sqrt{2 \pi}}\left|\frac{1}{\sqrt{M}}-\frac{1}{\sqrt{N}}\right| \cdot\left|b-\frac{1}{6}\left(\lambda_{1}+3 \lambda_{2}\right)\right|+R_{N, 1},
$$

otherwise

$$
\begin{aligned}
\sup _{x \in R} \mid F_{T_{N, M}^{*}}^{*} & (x)-F_{T_{N}}(x)\left|=\frac{1}{\sqrt{2 \pi}}\right| \frac{1}{\sqrt{M}}-\frac{1}{\sqrt{N}} \mid \\
\times \max & \left(\left|b-\frac{1}{6}\left(\lambda_{1}+3 \lambda_{2}\right)\right|, \frac{1}{3}\left|\lambda_{1}+3 \lambda_{2}\right| \exp \left(-\frac{3}{2}\left(1-\frac{2 b}{\lambda_{1}+3 \lambda_{2}}\right)\right)\right)+R_{N, 2},
\end{aligned}
$$

moreover, if $\frac{\lambda_{1}+6 b}{2 \lambda_{1}+3 \lambda_{2}} \leq-1$ or $2 \lambda_{1}+3 \lambda_{2}=0$, then

$$
\sup _{x \in R}\left|F_{T_{N, M}^{*}, S}^{*}(x)-F_{T_{N}, S}(x)\right|=\frac{1}{\sqrt{2 \pi}}\left|\frac{1}{\sqrt{M}}-\frac{1}{\sqrt{N}}\right| \cdot\left|b-\frac{1}{6}\left(\lambda_{1}+3 \lambda_{2}\right)\right|+R_{N, 3},
$$

otherwise

$$
\begin{aligned}
& \sup _{x \in R}\left|F_{T_{N, M}^{*}, S}^{*}(x)-F_{T_{N}, S}(x)\right|=\frac{1}{\sqrt{2 \pi}}\left|\frac{1}{\sqrt{M}}-\frac{1}{\sqrt{N}}\right| \\
& \quad \times \max \left(\left|b-\frac{1}{6}\left(\lambda_{1}+3 \lambda_{2}\right)\right|, \frac{1}{3}\left|2 \lambda_{1}+3 \lambda_{2}\right| \exp \left(-\frac{1}{2}\left(1+\frac{\lambda_{1}+6 b}{2 \lambda_{1}+3 \lambda_{2}}\right)\right)\right)+R_{N, 4},
\end{aligned}
$$

where for $j=1, \ldots, 4, R_{N, j}=o\left(N^{-1 / 2-q}+M^{-1 / 2} N^{-s}+M^{-1 / 2-p}\right)$, a.s. $[P]$, for every $s<a / 2$, $q<\min (a / 2,1 / 4)$ and $p$ satisfying $(2.20)$ as $\min (N, M) \rightarrow \infty$. 
It is evident from our results that the second order accuracy of the $M$ out of $N$ bootstrap approximations (i.e., the bound of order $o\left(N^{-1 / 2}\right)$ at the right-hand sides of relations in Corollary 2.2) can be achieved if and only if $M / N \rightarrow 1$ as $N \rightarrow \infty$, that is when $M=N+o(N)$. Moreover, if we assume a slightly stronger condition $M=N+O\left(N^{r}\right)$ with some $0 \leq r<1$, then

$$
\frac{1}{\sqrt{M}}-\frac{1}{\sqrt{N}}=O\left(N^{-1 / 2-(1-r)}\right) \text {. }
$$

This bound together with Corollary 2.2 directly implies the following result on the $M$ out of $N$ bootstrap.

Theorem 2.5. Suppose that condition $\left(C .1^{\prime}\right)$ is satisfied and let $M=N+O\left(N^{r}\right), 0 \leq r<1$. Then for every $p<\min (a / 2,1-r, 1 / 4)$

$$
\sup _{x \in R}\left|F_{T_{N, M}^{*}}^{*}(x)-F_{T_{N}}(x)\right|=o\left(N^{-1 / 2-p}\right),
$$

and

$$
\sup _{x \in R}\left|F_{T_{N, M}^{*}, S}^{*}(x)-F_{T_{N}, S}(x)\right|=o\left(N^{-1 / 2-p}\right),
$$

with probability one, as $N \rightarrow \infty$.

This result means that the $M$ out of $N$ bootstrap approximation to the trimmed mean (given by (2.1)) is more accurate than the standard normal approximation. In addition, we want to emphasize the fact, useful in statistical practice, that the bootstrap approximation is more accurate than the normal approximation not only in the case when $M=N$ (the naive bootstrap), but also in a more general situation when the size $M$ of the bootstrap sample differs from the size of the real data sample (but satisfies the condition $\left.M=N+O\left(N^{r}\right), r<1\right)$. Note that if condition $\left(C .1^{\prime}\right)$ is satisfied with $a \geq 1 / 2$ and $r \leq 3 / 4$, then $(2.28)-(2.29)$ provide the bounds of order $o\left(N^{-3 / 4+\varepsilon}\right)$ for every $\varepsilon>0$.

Let us now discuss why the trimmed mean given by $(2.1)$ is preferable for the bootstrap. Its computation is as easy as for $S_{N}$ (cf. (2.14)). Asymptotic expansions for $T_{N}$ and $S_{N}$ are identical in their skewness terms, but differ in the bias terms (cf. (2.13) and (2.15)). A similar remark applies to their bootstrap counterparts $T_{N, M}^{*}$ and $S_{N, M}^{*}$ (cf. Lemma 4.5).

Let the condition $M=N+O\left(N^{r}\right), r<1$, be satisfied, and note that the difference of two asymptotic expansions (for $S_{N}$ and for its bootstrap version $S_{N, M}^{*}$ ) contains the quantity $b_{[\cdot], N}-b_{[\cdot], N, M}$, which is of order $O\left(N^{-1 / 2}\right)$ in general, because of the presence of the differences of the fractional parts of $\nu N$ and $\nu M, \nu=\alpha, \beta$, which do not vanish when $N$ tends to infinity. Therefore, to establish our result on the second order accuracy of the bootstrap for $S_{N}$ (an analogue of Theorem 2.5) we need to take into account this bias contribution by a somewhat unpleasant correction of the location parameter in the definition of the $d f$ of the bootstrapped $S_{N}$ : for instance, we can put

$$
F_{S_{N, M}^{*}, S}^{*}(x)=P^{*}\left(\frac{M^{1 / 2}\left(S_{N, M}^{*}-T_{N}\right)}{(\beta-\alpha)^{-1} \sigma_{W, N}^{*}}-\frac{b_{[\cdot], N, M}-b_{[\cdot], N}}{\sigma_{W, N}^{*}} \leq x\right)
$$

(cf. second example, Section 3).

Another way to improve the accuracy of $M$ out of $N$ bootstrap for $S_{N}$ is to choose $M$ such that the fractional parts of $\nu N$ and $\nu M, \nu=\alpha, \beta$, are identical.

In the general case (i.e., without any bias correction) a Berry-Esseen type result (but not second order accuracy) can be achieved for the trimmed mean given by (2.14). Let $F_{S_{N}}(x), F_{S_{N}, S}(x)$ denote the functions $F_{T_{N}}(x), F_{T_{N}, S}(x)$, where $T_{N}$ is replaced by $S_{N}$ in their definition (cf. above), and $F_{S_{N, M}^{*}}^{*}(x)$, $F_{S_{N, M}^{*}, S}^{*}(x)$ are the functions $F_{T_{N, M}^{*}}^{*}(x)$ and $F_{T_{N, M}^{*}, S}^{*}(x)$ with $T_{N, M}^{*}$ replaced by $S_{N, M}^{*}$. The following theorem is a simple consequence of our expansions. 
Theorem 2.6. Suppose that conditions $\left(C .1^{\prime}\right)$ and $(C .2)$ are satisfied. Then

$$
\sup _{x \in R}\left|F_{S_{N, M}^{*}}^{*}(x)-F_{S_{N}}(x)\right|=O\left(\min (N, M)^{-1 / 2}\right)
$$

and

$$
\sup _{x \in R}\left|F_{S_{N, M}^{*}, S}^{*}(x)-F_{S_{N}, S}(x)\right|=O\left(\min (N, M)^{-1 / 2}\right)
$$

with probability one, as $\min (N, M) \rightarrow \infty$.

On the empirical EE, the $M$ out of $N$ bootstrap and extrapolation. Denote the functions appearing in the Edgeworth expansions $G_{N}(x)$ and $H_{N}(x)$ (cf. (2.12) and (2.22)) by

$$
B(x)=-\phi(x)\left[\frac{1}{6}\left(\lambda_{1}+3 \lambda_{2}\right)\left(x^{2}-1\right)+b\right],
$$

with $b$ as in (2.27), and

$$
B_{S}(x)=\phi(x)\left[\frac{1}{6}\left(\lambda_{1}\left(2 x^{2}+1\right)+3 \lambda_{2}\left(x^{2}+1\right)\right)-b\right]
$$

respectively.

Hence, we know that

$$
\begin{gathered}
F_{T_{N}}(x)=\Phi(x)+\frac{1}{\sqrt{N}} B(x)+R_{1, N}, \\
F_{T_{N}, S}(x)=\Phi(x)+\frac{1}{\sqrt{N}} B_{S}(x)+R_{2, N},
\end{gathered}
$$

where $R_{1, N}$ and $R_{2, N}$ are the remainder terms of the EE's. In [11] we estimate the unknown parameters appearing in $B(x)$ and $B_{S}(x)$ to obtain empirical EE's (EEE)

$$
\widehat{G}_{N}(x)=\Phi(x)+\frac{1}{\sqrt{N}} \widehat{B}(x) \quad \text { and } \quad \widehat{H}_{N}(x)=\Phi(x)+\frac{1}{\sqrt{N}} \widehat{B_{S}}(x) .
$$

We assume in [11] that (C.1) holds with $a=1$, and apply plug-in estimates for the moments and stepkernel estimates for the values of the density at the points $\xi_{\alpha}, \xi_{\beta}$ appearing in the Edgeworth correction terms for skewness and bias.

In practical applications, when the density is perhaps close to zero at the points $\xi_{\alpha}$, $\xi_{\beta}$, estimation of these small values may cause problems if the sample size $N$ is not very large. In this situation we might prefer the bootstrap procedure and to obtain second order accuracy, provided we perform simulations with $M=N+o(N)$ (cf. Theorem 2.5). This will of course require a lot of computation. Fortunately, however, we can considerably reduce the computation time by using the $M$ out of $N$ bootstrap (cf. $[7,23])$, when $\min (N, M) \rightarrow \infty$ and $M / N \rightarrow 0$, in conjunction with extrapolation.

Define the functions $B_{N}(x)$ and $B_{S, N}(x)$ to be the same as $B(x)$ and $B_{S}(x)$, with parameters $\lambda_{1}, \lambda_{2}$, $\alpha, \beta, \sigma_{W}, f\left(\xi_{\alpha}\right)$, and $f\left(\xi_{\beta}\right)$ replaced by $\lambda_{1, N}, \lambda_{2, N}, \alpha_{N}, \beta_{N}, \sigma_{W, N}, f\left(\xi_{\alpha N: N}\right)$, and $f\left(\xi_{\beta N: N}\right)$. Then, with $M \ll N$ we have the bootstrap version of (2.30):

$$
\begin{gathered}
F_{T_{N, M}^{*}}^{*}(x)=\Phi(x)+\frac{1}{\sqrt{M}} B_{N}(x)+R_{1, N, M}, \\
F_{T_{N, M}^{*}, S}^{*}(x)=\Phi(x)+\frac{1}{\sqrt{M}} B_{S, N}(x)+R_{2, N, M},
\end{gathered}
$$

where $R_{j, N, M}, j=1,2$, are of order $o\left(M^{-1 / 2-p}\right)$ for every $p<\min (a / 2,1 / 4)$, with probability one. Note that the functions $B_{N}(x)$ and $B_{S, N}(x)$ depend on $F, N$, but not on $M$ (and here it is crucial that we consider $T_{N}$ given by (2.1) instead of $S_{N}$ given by (2.14)). 
Therefore relations (2.31) allow us to estimate these functions with an error of order $o\left(M^{-p}\right)$ with probability one. The next step is to employ extrapolation for $M=N$, i.e., we substitute these estimates in the expressions for $G_{N, N}(x)$ and $H_{N, N}(x)$ to obtain the estimates

$$
\widehat{F}_{T_{N, N}^{*}}^{*}(x)=\Phi(x)+\frac{1}{\sqrt{N}} \widehat{B}_{N}(x), \quad \widehat{F}_{T_{N, N}^{*}, S}^{*}(x)=\Phi(x)+\frac{1}{\sqrt{N}} \widehat{B}_{S, N}(x),
$$

with an error of order $o\left(N^{-1 / 2} M^{-p}\right)$, implying second order accuracy. Hence we obtain our first main result for the $M$ out of $N$ bootstrap, whenever $M$ is of smaller order than $N$ (cf. [7, 23]).

Theorem 2.7. Suppose that condition $\left(C .1^{\prime}\right)$ is satisfied and $M / N \rightarrow 0$ as $M \rightarrow \infty$. Then

$$
\begin{aligned}
\sup _{x \in R}\left|\widehat{F}_{T_{N}^{*}}^{*}(x)-F_{T_{N}}(x)\right| & =o\left(N^{-1 / 2} M^{-p}\right), \\
\sup _{x \in R}\left|\widehat{F}_{T_{N, N}^{*}, S}^{*}(x)-F_{T_{N}, S}(x)\right| & =o\left(N^{-1 / 2} M^{-p}\right),
\end{aligned}
$$

for every $p<\min (a / 2,1 / 4)$, with probability one, as $M \rightarrow \infty$.

In particular, for $a \geq 1 / 2$ and with $M=\left[N^{q}\right], 0<q<1$, we obtain an $M$ out of $N$ bootstrap approximation with error of order $o\left(N^{-(1 / 2+q / 4-\varepsilon)}\right)$, for every $\varepsilon>0$. Hence, as in Theorem 2.5 , we obtain second order accuracy of the bootstrap, but now with reduced computational time. We also note in passing that extrapolation for the trimmed mean given by $S_{N}$ (cf. (2.14)) only yields a slow rate of order $O\left(N^{-1 / 2}\right)$ (cf. Theorem 2.6). In other words: improvement over the normal approximation is not possible here for $S_{N}$.

The next step is to improve the $M$ out of $N$ bootstrap by extrapolation using a simple linear regression model as proposed in [23] (cf. also [7]). In our case we need to estimate one parameter $B=B_{N}(x)$ (or $B=B_{S, N}(x)$ for the case of Studentized statistic). Take $M_{k}=\left[t_{k} N^{q}\right], k=1, \ldots, K$, where $K \geq 1$ is integer, $0<q<1$, and $t_{1}<t_{2}<\cdots<t_{K}$ are fixed. Perform the bootstrap procedure $K$ times with $M=M_{k}, k=1, \ldots, K$. We obtain

$$
Y_{k}(x)=F_{T_{N, M_{k}}^{*}}^{*}(x)-\Phi(x)=M_{k}^{-1 / 2} B_{N}(x)+O\left(N^{-q(1 / 2+p)}\right)
$$

with $p<\min (a / 2,1 / 4)$. Then, we estimate $B_{N}(x)$ in formula (2.33) by viewing $B_{N}(x)$, for fixed $x$, as a parameter in a linear regression, with error term $o\left(N^{-q(1 / 2+p)}\right)$. Applying least squares we obtain the estimate

$$
\widehat{B}_{N}(x)=\sum_{k=1}^{K} Y_{k}(x) M_{k}^{-1 / 2} / \sum_{k=1}^{K} M_{k}^{-1}=N^{d / 2} \sum_{k=1}^{K} t_{k}^{-1 / 2} Y_{k}(x) / \sum_{k=1}^{K} t_{k}^{-1},
$$

and similarly for $\widehat{B}_{S, N}(x)$ with $Y_{k}(x)=F_{T_{N, M_{k}}^{*}, S}^{*}(x)-\Phi(x)$. It is evident that the order of the error of this estimates is $o\left(N^{-q p}\right)$, and substituting these estimates in the formulas (2.32) gives the order $o\left(N^{-(1 / 2+q p)}\right)$ for the error of the approximations (2.32). Thus, we obtain our second result for the $M$ out of $N$ bootstrap in conjunction with extrapolation, whenever $M$ is of smaller order than $N$.

Theorem 2.8. Suppose that condition $\left(C .1^{\prime}\right)$ is satisfied and estimates $\widehat{B}_{N}(x)$ and $\widehat{B}_{S, N}$ in $(2.32)$ are given by (2.34). Then for every $p<\min (a / 2,1 / 4)$

$$
\begin{array}{r}
\sup _{x \in R}\left|\widehat{F}_{T_{N}^{*}}^{*}(x)-F_{T_{N}}(x)\right|=o\left(N^{-(1 / 2+q p)}\right), \\
\sup _{x \in R}\left|\widehat{F}_{T_{N, N}^{*}, S}^{*}(x)-F_{T_{N}, S}(x)\right|=o\left(N^{-(1 / 2+q p)}\right),
\end{array}
$$

with probability one, as $N \rightarrow \infty$. 
We can expect some improvement in comparison with the previous method $(K=1)$ due to additional information. We present simulations on $M$ out of $N$ bootstrap in conjunction with extrapolation in Section 3.

The Naive Bootstrap. Consider the special case $M=N$, i.e., the so-called 'naive bootstrap'. We apply our main results and discuss bootstrapping for both versions of trimmed mean.

(I) Bootstrapping for $T_{N}$. Consider the trimmed mean $T_{N}$ given by (2.1). The following consequence of Theorem 2.5 yields the second order accuracy of the naive bootstrap for the normalized and Studentized $T_{N}$.

Theorem 2.9. Suppose that condition $\left(C .1^{\prime}\right)$ is satisfied. Then for every $p<\min (a / 2,1 / 4)$

$$
\begin{aligned}
\sup _{x \in R}\left|F_{T_{N}^{*}}^{*}(x)-F_{T_{N}}(x)\right| & =o\left(N^{-1 / 2-p}\right), \\
\sup _{x \in R} \mid F_{T_{N, N}^{*}}^{*}, S & (x)-F_{T_{N}, S}(x) \mid=o\left(N^{-1 / 2-p}\right),
\end{aligned}
$$

with probability one, as $N \rightarrow \infty$.

Note that the extreme terms, containing fractional parts of $\alpha N$ and $\beta N$ in the definition of the $d f$ of the bootstrap counterpart of $T_{N}$, can be omitted when $M=N$. Indeed, by Lemma 4.2 the quantity $N^{-1 / 2}\left(X_{[\alpha N]+1: N}^{*}-X_{[\alpha N]+1: N}\right)$ in our smoothness condition is of order $O\left((\log N)^{1 / 2} / N\right)$, with $P^{*}$ probability $1-O\left(N^{-c}\right)$, for every $c>0$, a.s. $[P]$, because the expression at the r.h.s. of (4.8) (with $M=N$ and function $G(x)=x)$ is of order $O\left((\log N / N)^{1 / 2}\right)$. The same is valid for $N^{-1 / 2}\left(X_{[\beta N]+1: N}^{*}\right.$ $\left.X_{[\beta N]+1: N}\right)$. Therefore, for the $d f$ of the bootstrapped trimmed mean we have

$$
\begin{aligned}
F_{T_{N, N}^{*}}^{*}(x) & =P^{*}\left(\frac{N^{1 / 2}\left(T_{N, N}^{*}-T_{N}\right)}{(\beta-\alpha)^{-1} \sigma_{W, N}} \leq x\right) \\
& =P^{*}\left(N^{-1 / 2} \sigma_{W, N}^{-1}\left(\sum_{i=[\alpha N]+1}^{[\beta N]} X_{i: N}^{*}-\sum_{i=[\alpha N]+1}^{[\beta N]} X_{i: N}\right) \leq x+R_{N}\right),
\end{aligned}
$$

where $R_{N}$ is a quantity of order $O\left((\log N)^{1 / 2} / N\right)$, with $P^{*}$-probability $1-O\left(N^{-c}\right)$, for every $c>0$, a.s. $[P]$. Since the derivative $G_{N, N}^{\prime}$ of the one-term EE is bounded uniformly, the term $R_{N}$ can be deleted, because it adds to the Edgeworth expansion $G_{N, N}(x)$ a quantity contributing to a remainder term. Similarly, the $d f$ of the Studentized bootstrap trimmed mean $F_{T_{N, N}^{*}, S}^{*}(x)$ can be reduced to

$$
P^{*}\left(N^{-1 / 2} \sigma_{W, N}^{*-1}\left(\sum_{i=[\alpha N]+1}^{[\beta N]} X_{i: N}^{*}-\sum_{i=[\alpha N]+1}^{[\beta N]} X_{i: N}\right) \leq x\right)
$$

(II) Bootstrapping for $S_{N}$. Next consider the trimmed mean given by $S_{N}$ (cf. (2.14)). Define $d f$ 's of the normalized and Studentized $S_{N}$ by

$$
F_{S_{N}}(x)=P\left(\frac{N^{1 / 2}\left(S_{N}-\mu(\alpha, \beta)\right)}{(\beta-\alpha)^{-1} \sigma_{W}} \leq x\right) \quad \text { and } \quad F_{S_{N}, S}(x)=P\left(\frac{N^{1 / 2}\left(S_{N}-\mu(\alpha, \beta)\right)}{(\beta-\alpha)^{-1} \sigma_{W, N}} \leq x\right)
$$

(cf. [11]). The corresponding asymptotic expansions are $G_{N}(x)$ and $H_{N}(x)$, where the bias term $b_{N}$ is replaced by $d_{N}$ (cf. Lemma 4.5). The bootstrap counterpart of $S_{N}$ is defined by

$$
S_{N, N}^{*}=\frac{1}{([\beta N]-[\alpha N])} \sum_{i=\alpha N+1}^{\beta N} X_{i: N}^{*} .
$$


The $d f$ 's of the normalized and Studentized $S_{N, N}^{*}$ are defined by

$$
F_{S_{N, N}^{*}}^{*}(x)=P^{*}\left(\frac{N^{1 / 2}\left(S_{N, N}^{*}-T_{N}\right)}{(\beta-\alpha)^{-1} \sigma_{W, N}} \leq x\right) \quad \text { and } \quad F_{S_{N, S}^{*}, S}^{*}(x)=P^{*}\left(\frac{N^{1 / 2}\left(S_{N, N}^{*}-T_{N}\right)}{(\beta-\alpha)^{-1} \sigma_{W, M}^{*}} \leq x\right),
$$

the corresponding bootstrap asymptotic expansions are $G_{N, N}(x)$ and $H_{N, N}(x)$ (with bias term $d_{N, N}$ (cf. (2.26)). Because our smoothness condition $\left(C .1^{\prime}\right)$ implies that the term $b_{[\cdot], N}-b_{[\cdot], N, N}$ (cf. (2.15), (2.26) with $M=N$ ) is of negligible order and contributes to the remainder term, we obtain the second order accuracy of the naive bootstrap for the r.v. $S_{N}$ (cf. [13] for a similar result under somewhat stronger conditions).

Theorem 2.10. Suppose that condition $\left(C .1^{\prime}\right)$ is satisfied. Then for every $p<\min (a / 2,1 / 4)$

$$
\begin{array}{r}
\sup _{x \in R}\left|F_{S_{N}^{*}}^{*}(x)-F_{S_{N}}(x)\right|=o\left(N^{-1 / 2-p}\right), \\
\sup _{x \in R}\left|F_{S_{N}^{*}, S}^{*}(x)-F_{S_{N}, S}(x)\right|=o\left(N^{-1 / 2-p}\right),
\end{array}
$$

with probability one, as $N \rightarrow \infty$.

To conclude this section, we note that a completely different way of approximating $F_{S_{N}}$ and $F_{S_{N}, S}$ is to use saddlepoint approximations. These approximations will typically work better in the tail of the distribution in comparison with the Edgeworth expansions and bootstrap approximations considered in the present paper. Only very recently these saddlepoint expansions were established for the trimmed mean and the Studentized trimmed mean. We refer to [16] for more details.

\section{SIMULATIONS}

In this section we illustrate our results on Edgeworth expansions, bootstrap approximations, and our previous results on the empirical Edgeworth expansion in [11] by simulations.

We consider the case of the normalized trimmed mean as well as the case of the Studentized one, and we look at both $T_{N}$ given in (2.1) and $S_{N}$ as in (2.14) (cf. Section 2). Monte Carlo simulations were performed for the following distributions: standard exponential and a mixture of two normal distributions. To compute the distribution functions $F_{T_{N}}, F_{S_{N}}$ (of the normalized statistics) and $F_{T_{N}, S}, F_{S_{N}, S}$ (of their Studentized versions) we use Monte Carlo procedure with $10^{6}$ samples.

The values of one-term Edgeworth expansions were computed in each case by our formulas for $G_{N}(x)$ and $H_{N}(x)$. To get the values of the empirical Edgeworth expansions we need to estimate the parameters of the first term of the EE. We use plug-in estimates for the moments and quantiles, and kernel estimates for the values of the density at $\xi_{\alpha}, \xi_{\beta}$, taking simple step-kernel with $N^{-1 / 4}$ as the width of the step (in the same way as in [11]). For computation of the $d f$ 's of the bootstrapped trimmed mean: $F_{T_{N, M}^{*}}^{*}, F_{S_{N, M}^{*}}^{*}$ (normalized) and $F_{T_{N, M}^{*}, S}^{*}, F_{S_{N, M}^{*}, S}^{*}$ (Studentized) (cf. Section 2) we also apply a Monte Carlo procedure using $10^{5}$ samples. We compute the differences of the various approximations to the $d f$ of the trimmed mean and its true $d f$, and plot these differences in our figures.

In Fig. 1 we present our results for the Studentized trimmed mean $T_{N}$ given by (2.1), where $\alpha=1-\beta=0.25$, and as underlying distribution we take a mixture of two normal laws:

$$
F(x)=p \Phi\left(\left(x-a_{1}\right) / \sigma_{1}\right)+(1-p) \Phi\left(\left(x-a_{2}\right) / \sigma_{2}\right) .
$$

Here $a_{1}=0, \sigma_{1}=0.5, a_{2}=2, \sigma_{2}=1, p=0.6$, sample size $N=45$ and $M=N$ (the naive bootstrap). It can be seen that bootstrap is the best and all the second order approximations (EE, EEE and bootstrap) are better than the normal one, as one would expect.

Our second example deals with the $M$ out of $N$ bootstrap $(M \neq N)$. The simulations were performed for the standard exponential distribution. Here we consider $S_{N}$ (cf. (2.14), Section 2) with $\alpha=1-\beta=$ 0.15. In Fig. 2 we plot the differences for the normal $d f$, EE and bootstrap with $M=N, M=2 N$ and 


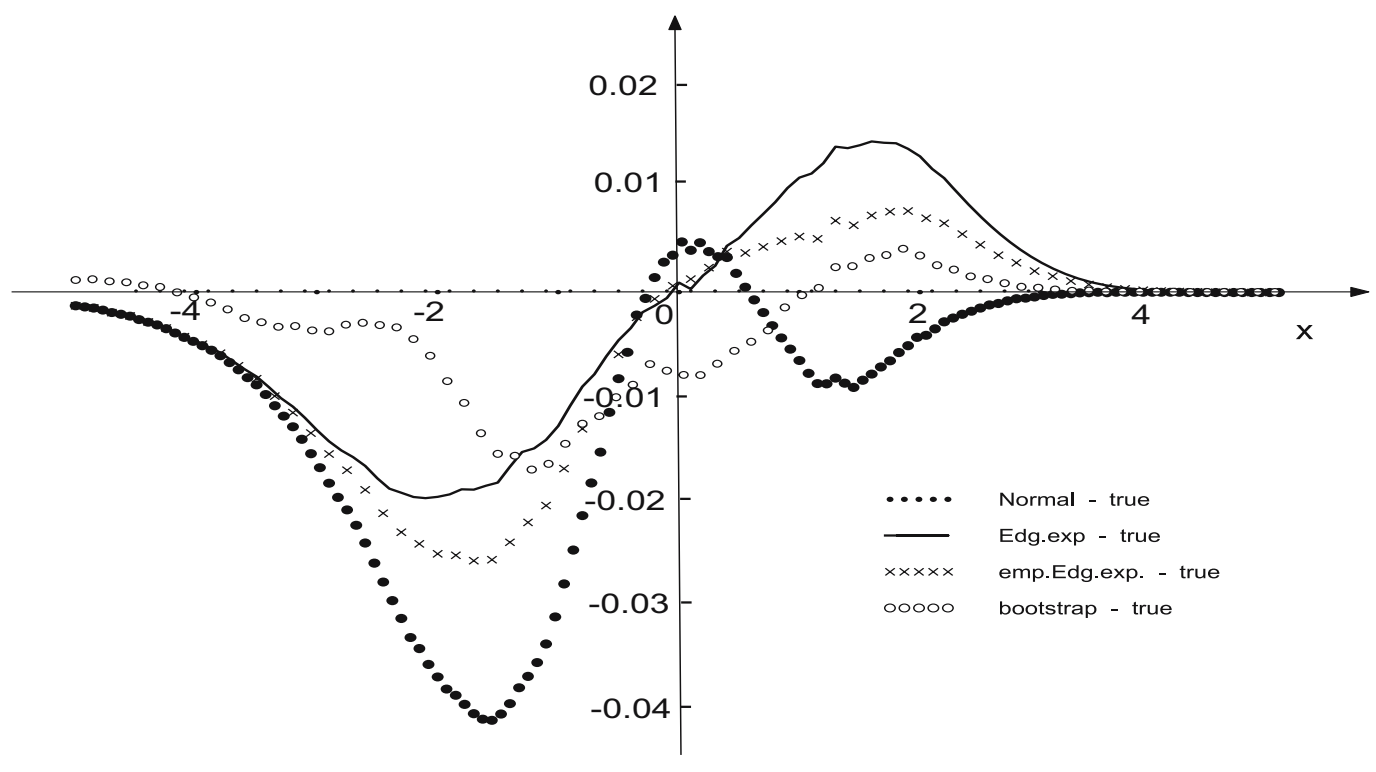

Fig. 1. $T_{N}$, Studentized, mixture of two normal distributions, $N=45$

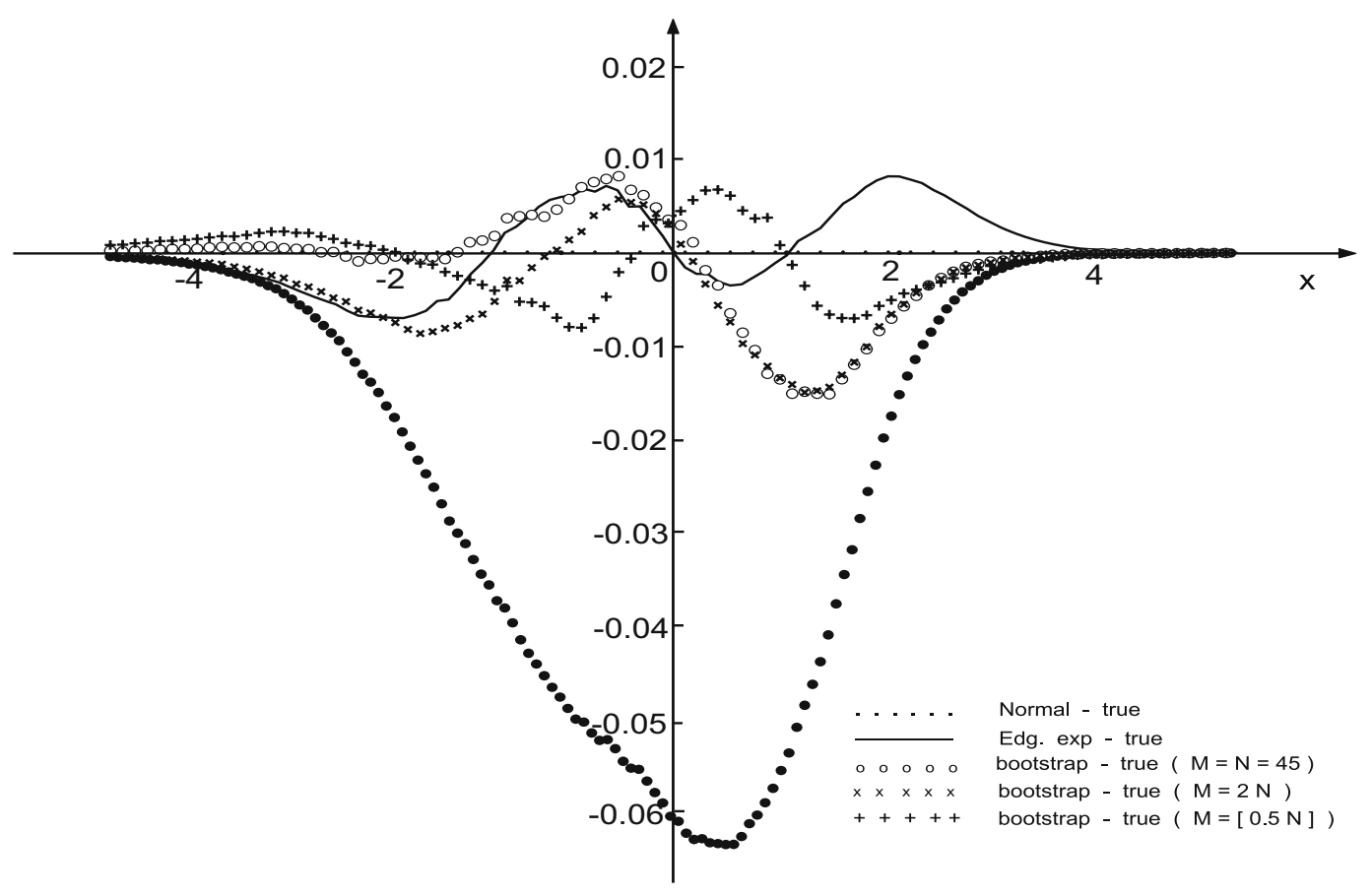

Fig. 2. $S_{N}$, Studentized, standard exponential distribution, $N=45$ 
$M=[0.5 N]$, where $N=45$. To obtain bootstrap approximations in this case we performed the Monte Carlo procedure for the $d f$ given by

$$
F_{S_{N, M}^{*}, S}^{*}(x)=P^{*}\left(\frac{M^{1 / 2}\left(S_{N, M}^{*}-T_{N}\right)}{(\beta-\alpha)^{-1} \sigma_{W, M}^{*}}-\frac{b_{[\cdot], N, M}}{\sigma_{W, M}^{*}}+\frac{\hat{b}_{[\cdot], N}}{\sigma_{W, N}} \leq x\right),
$$

with $b_{[\cdot], N, M}$ as in (2.26), $\hat{b}_{[\cdot], N}$ the estimator for $b_{[\cdot], N}($ cf. $(2.15))$,

$$
\hat{b}_{[\cdot], N}=-\frac{1}{\sqrt{N}}\left\{-(\alpha N-[\alpha N])\left(T_{N}-\xi_{\alpha N: N}\right)+(\beta N-[\beta N])\left(T_{N}-\xi_{\beta N: N}\right)\right\} .
$$

We complete this section with an example involving extrapolation. Simulations were performed with standard exponential distribution for the Studentized trimmed mean $T_{N}$ given by (2.1), where $N=81$, $\alpha=0.25$ and $\beta=0.85$. We use the regression model of extrapolation (cf. Section 1, cf. also [7]) to estimate the first term of the EE. First we compute the values of $d f$ of the bootstrapped Studentized statistic $F_{T_{N, M}^{*}, S}^{*}(x)$ three times with sample sizes $M_{k}=t_{k} \sqrt{N}, t_{k}=1,2,3$, that is $M_{1}=9, M_{2}=18$, $M_{3}=27$, then we estimate the function $B_{S}(x)$ as a parameter in regression by the 'least squares estimate'

$$
\widehat{B}_{S}(x)=\sum_{k=1}^{3}\left(\left(F_{T_{N, M_{k}}^{*}, S}^{*}(x)-\Phi(x)\right) M_{k}^{-1 / 2}\right) / \sum_{k=1}^{3} M_{k}^{-1}
$$

(cf. (2.34)), and substitute it to the formula for the one term EE. As the result we obtain an estimate $\widehat{F}_{T_{N}, S}=\Phi(x)+\frac{1}{\sqrt{N}} \widehat{B}_{S}(x)$ of the $d f$ of the Studentized statistic $T_{N}$.

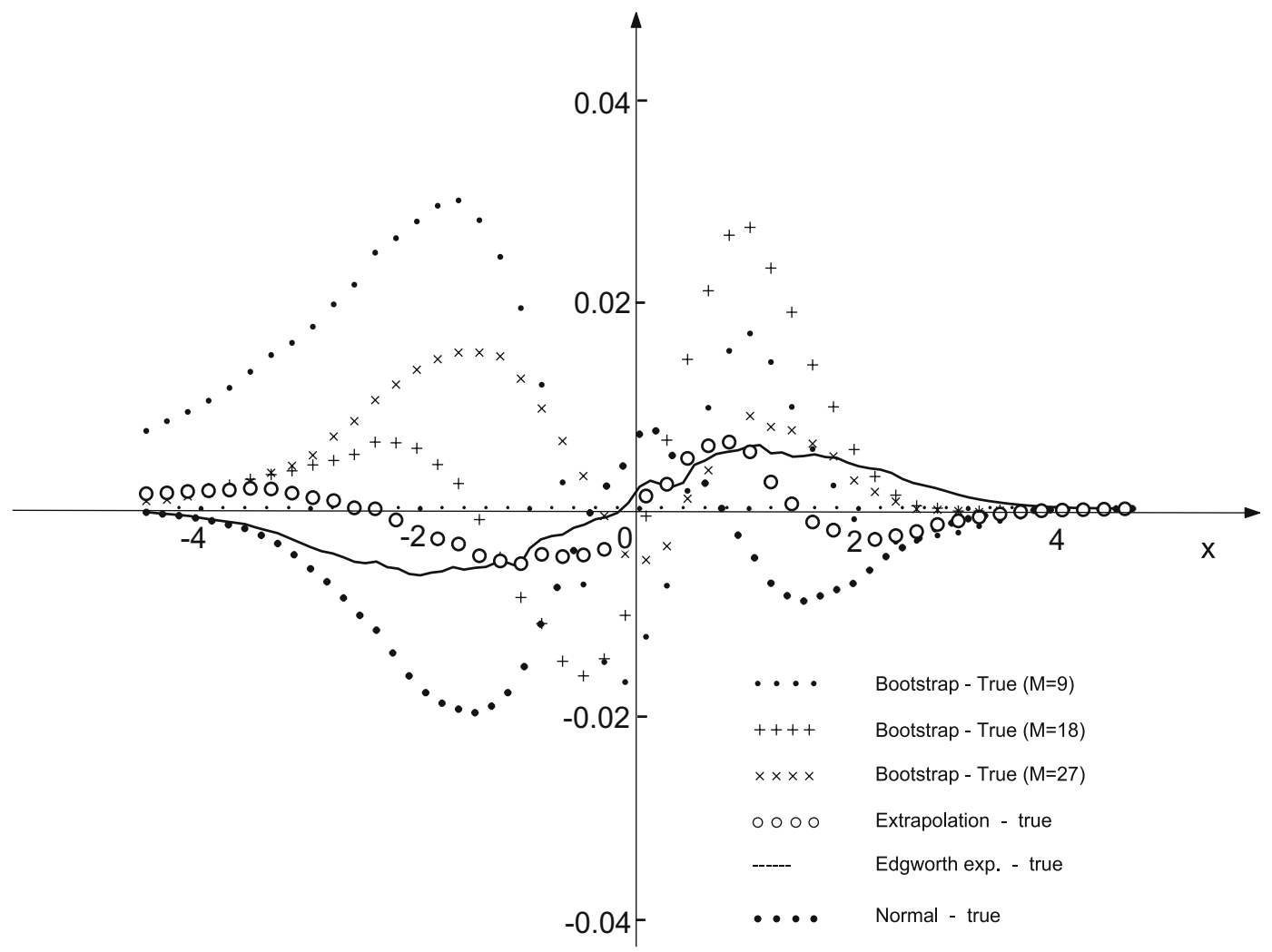

Fig. 3. $T_{N}$, Studentized, Extrapolation, $N=81, M_{1}=9, M_{2}=18, M_{3}=27$

The results of our simulations are presented in Fig. 3. We plot the differences between $F_{T_{N, M_{k}}^{*}, S}^{*}(x)$ and the true $d f(k=1,2,3)$ as well as the difference between the result of extrapolation $\widehat{F}_{T_{N}, S}(x)$ and 
the true $d f$. We see that extrapolation gives the best approximation in our example. Also note that the accuracy of the $M$ out of $N$ bootstrap gets better when $M$ becomes larger. This is of course as one would expect. Simulations show that both $\mathrm{EE}$ and the naive bootstrap extrapolation give second order approximation and achieve this with reduced computational time as compared with the naive bootstrap. We also refer to Fig. 2 of [7] for a similar result for the trimmed mean.

\section{SOME LEMMAS}

In this section we state and prove a few lemmas, which we will need in our proofs.

Bahadur's type results. Let $G$ be a function defined and differentiable with derivative $g$ in a neighborhood of $\xi_{\alpha}=F^{-1}(\alpha), 0<\alpha<1$.

The following lemma is an extension of Lemma 3.1 in [11] (cf. also [2] and Theorem 6.3.1 in Reiss [22]).

Lemma 4.1. Suppose that $f=F^{\prime}$ exists in a neighborhood of $\xi_{\alpha}$ and $f\left(\xi_{\alpha}\right)>0$. In addition, assume that the functions $f$ and $g$ satisfy a Hölder condition of order $a>0$ at the point $\xi_{\alpha}$. Then

$$
G\left(\xi_{\alpha N: N}\right)-G\left(\xi_{\alpha}\right)=-\left[F_{N}\left(\xi_{\alpha}\right)-F\left(\xi_{\alpha}\right)\right] g\left(\xi_{\alpha}\right) / f\left(\xi_{\alpha}\right)+R_{N},
$$

where $P\left(\left|R_{N}\right|>N^{-1 / 2-p}\right)=o\left(N^{-c}\right)$, for any $c>0$ and every $p<\min (a / 2,1 / 4)$, as $N \rightarrow \infty$.

Proof. Write (cf. (4.1))

$$
R_{N}=G\left(\xi_{\alpha N: N}\right)-G\left(\xi_{\alpha}\right)+\frac{N_{\alpha}-\alpha N}{N} g\left(\xi_{\alpha}\right) / f\left(\xi_{\alpha}\right),
$$

where $N_{\alpha}=\sharp\left\{i: X_{i} \leq \xi_{\alpha}\right\}$. Let $U_{1}, \ldots, U_{N}$ be independent, uniformly $(0,1)$ distributed r.v.'s, and let $U_{1: N} \leq \cdots \leq U_{N: N}$ denote the corresponding order statistics. Define $F_{N, u}(x)=N^{-1} \sharp\left\{i: U_{i} \leq x\right\}$, $0<x \leq 1$, the empirical $d f$ and $v_{\alpha N: N}=F_{N, u}^{-1}(\alpha)$ the corresponding $\alpha$-quantile. Note that $\xi_{\alpha N: N}$ and $N_{\alpha}$ are distributed as $F^{-1}\left(v_{\alpha N: N}\right)$ and $N_{\alpha, u}=\sharp\left\{i: U_{i} \leq \alpha\right\}$. Therefore $R_{N}$ is distributed as

$$
G\left(F^{-1}\left(v_{\alpha N: N}\right)\right)-G\left(F^{-1}(\alpha)\right)+\frac{N_{\alpha, u}-\alpha N}{N} g\left(\xi_{\alpha}\right) f\left(\xi_{\alpha}\right) .
$$

The latter quantity equals

$$
\left(v_{\alpha N: N}-\alpha\right) g\left(\xi_{\alpha}\right) / f\left(\xi_{\alpha}\right)+R_{N, 1}+\frac{N_{\alpha, u}-\alpha N}{N} g\left(\xi_{\alpha}\right) / f\left(\xi_{\alpha}\right),
$$

where $\left|R_{N, 1}\right| \leq C_{a}\left|v_{\alpha N: N}-\alpha\right|^{1+a}$, and $C_{a}$ is the Hölder constant of the function $g\left(F^{-1}(u)\right) / f\left(F^{-1}(u)\right)$ at the point $\alpha$ (we may neglect here the event when $v_{\alpha N: N}$ does not belong to the neighborhood, where condition (C.1) is satisfied). By Theorem 6.3.1 of Reiss [22] (cf. also Lemma 3.1 in [11]) we have

$$
v_{\alpha N: N}-\alpha=-\frac{N_{\alpha, u}-\alpha N}{N}+R_{N, u}
$$

where $R_{N, u}$ is the remainder of Bahadur's order, i.e.,

$$
P\left(\left|R_{N, u}\right|>A(\log N / N)^{3 / 4}\right)=O\left(N^{-c}\right),
$$

for every $c>0$ with some $A>0$, independent of $N$. This implies that the quantity (4.4) is equal to $R_{N, 1}+R_{N, 2}$, where $R_{N, 2}$ satisfies (4.6), and for $R_{N, 1}$ we have

$$
P\left(\left|R_{N, 1}\right|>(A \log N / N)^{(a+1) / 2}\right) \leq P\left(\left|v_{\alpha N: N}-\alpha\right|>C_{a}^{-1 /(a+1)}(A \log N / N)^{1 / 2}\right),
$$

and by Bernstein's inequality the last probability is $O\left(N^{-c}\right)$, provided $A>2 c \alpha(1-\alpha) C_{a}^{2 /(a+1)}$. Moreover, our argument also implies that for any $p<\min (a / 2,1 / 4)$ we have $\left|R_{N, j}\right|<N^{-1 / 2-p} \quad(j=1,2)$ with probability $1-o\left(N^{-c}\right)$ for every $c>0$. This together with (4.2)-(4.3) implies (4.1). The lemma is proved. 
Remark 4.1. It follows from the proof of Lemma 4.1 that the remainder term in (4.1) is a sum of two terms: $R_{N}=R_{N, 1}+R_{N, 2}$, where $\left|R_{N, 1}\right| \leq A(\log N / N)^{3 / 4}$ and $\left|R_{N, 2}\right| \leq B(\log N / N)^{1 / 2+a / 2}$, with probability $1-O\left(N^{-c}\right)$, for every $c>0$, where $A>0$ is a constant depending on $\alpha$ and $c$, and $B>0$ is a constant depending on $\alpha, c$, and $F$, but not on $N$. Hence our bound for $R_{N}$ is in fact slightly better than the one in Lemma 4.1.

Our second lemma is concerned with the validity of Bahadur's representation for an $M$ out of $N$ bootstrap quantile.

Lemma 4.2. Suppose that $f=F^{\prime}$ exists in a neighborhood of $\xi_{\alpha}$ and $f\left(\xi_{\alpha}\right)>0$. In addition assume that the functions $f$ and $g$ satisfy the uniform Hölder condition of order $a>0$ in $a$ neighborhood of $\xi_{\alpha}$, and that condition (C.2) holds true.

Then

$$
G\left(\xi_{\alpha M: M}^{*}\right)-G\left(\xi_{\alpha N: N}\right)=-\left[F_{M}^{*}\left(\xi_{\alpha N: N}\right)-F_{N}\left(\xi_{\alpha N: N}\right)\right] g\left(\xi_{\alpha N: N}\right) / f\left(\xi_{\alpha N: N}\right)+R_{M}^{*},
$$

where $P^{*}\left(\left|R_{M}^{*}\right|>M^{-1 / 2-p}\right)=o\left(M^{-c}\right)$ for every $p$ satisfying (2.20), with probability one, as $\min (N, M) \rightarrow \infty$.

We relegate the proof of Lemma 4.2 to Section 7.

Remark 4.2. Note that if $a \geq 1 / 2$, then the remainder term $R_{N}$ in (4.1) is of the order $o\left(N^{-3 / 4+\varepsilon}\right)$ for every $\varepsilon>0$, and if additionally $d=1$, then the remainder term $R_{M}^{*}$ in (4.8) is of order $o\left(M^{-3 / 4+\varepsilon}\right)$ with probability one, for every $\varepsilon>0$.

The following lemma represents a refined version of Lemma 3.2 from [11].

Lemma 4.3. Suppose that the conditions of Lemma 4.1 hold. Then

$$
\int_{\xi_{\alpha N: N}}^{\xi_{\alpha}}\left(G(x)-G\left(\xi_{\alpha}\right)\right) d F_{N}(x)=-\frac{1}{2}\left[F_{N}\left(\xi_{\alpha}\right)-F\left(\xi_{\alpha}\right)\right]^{2} g\left(\xi_{\alpha}\right) / f\left(\xi_{\alpha}\right)+R_{N},
$$

where $P\left(\left|R_{N}\right|>N^{-1-p}\right)=o\left(N^{-c}\right)$ for any $c>0$ and every $p<\min (a / 2,1 / 4)$, as $N \rightarrow \infty$.

Remark 4.3. Relation (4.9) is a direct consequence of integrating the Bahadur-Kiefer process (cf. [20]) in the interval $\left[\xi_{\alpha N: N}, \xi_{\alpha}\right)$. As we need upper bounds 'in probability', whereas in [24]'almost sure' results are derived, our smoothness conditions are slightly weaker than the ones in [24] and [20]. For this reason we include a short proof of (4.9). Relation (4.9) parallels the assertions (3.2) and (3.3) in [13]; note that our smoothness condition on the density $f$ is slightly weaker than the one used in [13]. The factors $(1-\alpha)^{-1}$ and $(1-\beta)^{-1}$ appearing in [13] are superfluous.

Proof. Let us adopt the following notation: for any integer $k$ and $m$ let

$$
\sum_{i=k}^{m}(.)_{i}=\operatorname{sign}[m-k] \sum_{i=k \wedge m}^{k \vee m}(.)_{i} .
$$

Then we can rewrite the integral on the 1.h.s. of (4.9) as $N^{-1} \sum_{i=k_{\alpha}}^{N_{\alpha}}\left(G\left(X_{i: N}\right)-G\left(\xi_{\alpha}\right)\right)$, where as in the proof of Lemma 4.1 $N_{\alpha}=\sharp\left\{i: X_{i} \leq \xi_{\alpha}\right\}$ and $k_{\alpha}=\alpha_{N} N$ is the index of the order statistic corresponding to the $\alpha$-th quantile of the sample $X_{1}, \ldots, X_{N}$. Now we need to estimate the remainder (cf. (4.9)):

$$
R_{N}=\frac{1}{2} \frac{\left(N_{\alpha}-\alpha N\right)^{2}}{N^{2}} g\left(\xi_{\alpha}\right) / f\left(\xi_{\alpha}\right)+\frac{1}{N} \sum_{i=k_{\alpha}}^{N_{\alpha}}\left(G\left(X_{i: N}\right)-G\left(\xi_{\alpha}\right)\right) .
$$


Similarly to the proof of Lemma 4.1, we find that $R_{N}$ is distributed as

$$
\frac{1}{2} \frac{\left(N_{\alpha, u}-\alpha N\right)^{2}}{N^{2}} g\left(\xi_{\alpha}\right) / f\left(\xi_{\alpha}\right)+\frac{1}{N} \sum_{i=k_{\alpha}}^{N_{\alpha, u}}\left(G\left(F^{-1}\left(U_{i: N}\right)\right)-G\left(F^{-1}(\alpha)\right)\right) .
$$

The second term of (4.11) equals

$$
-\frac{1}{2} \frac{\left(N_{\alpha, u}-\alpha N\right)^{2}}{N^{2}} g\left(\xi_{\alpha}\right) / f\left(\xi_{\alpha}\right)+R_{N, 1}+R_{N, 2}
$$

where $P\left(\left|R_{N, 1}\right|>A(\log N / N)^{5 / 4}\right)=O\left(N^{-c}\right)$ for every $c>0$ and some $A>0$, independent of $N$ (cf. Lemma 3.2 in [11]), and

$$
R_{N, 2}=N^{-1} \sum_{i=k_{\alpha}}^{N_{\alpha, u}}\left[\left(G \circ F^{-1}\right)^{\prime}\left(\alpha+\theta_{i}\left(U_{i: N}-\alpha\right)\right)-\left(G \circ F^{-1}\right)^{\prime}(\alpha)\right]\left(U_{i: N}-\alpha\right),
$$

where $\left|\theta_{i}\right| \leq 1$. The Hölder condition directly implies that

$$
\begin{aligned}
\left|R_{N, 2}\right| & \leq \frac{1}{N} \sum_{i=k_{\alpha} \wedge N_{\alpha, u}}^{k_{\alpha} \vee N_{\alpha, u}} C_{a}\left|U_{i: N}-\alpha\right|^{1+a} \\
& \leq \frac{C_{a}\left|N_{\alpha, u}-k_{\alpha}\right|}{N} \max \left(\left|v_{\alpha N: N}-\alpha\right|^{1+a},\left|U_{N_{\alpha, u}: N}-\alpha\right|^{1+a}\right) .
\end{aligned}
$$

Bernstein's inequality yields that $\left|N_{\alpha, u}-k_{\alpha}\right|<A_{1}(N \log N)^{1 / 2},\left|v_{\alpha N: N}-\alpha\right|<A_{2}(\log N / N)^{1 / 2}$, and $\left|U_{N_{\alpha, u}: N}-\alpha\right|<A_{3}(\log N / N)$ with probability $1-O\left(N^{-c}\right)$, for every $c>0$ and some $A_{i}, i=1,2,3$, independent of $N$ (cf. Lemma 3.2 in [11]). This together with (4.13) ensures that

$$
P\left(\left|R_{N, 2}\right|>A_{4}(\log N / N)^{1+a / 2}\right)=O\left(N^{-c}\right),
$$

as $N \rightarrow \infty$ for every $c>0$ with some $A_{4}>0$, independent of $N$. Relations (4.10)-(4.12) and (4.14) imply (4.9) for every $p<\min (a / 2,1 / 4)$ and $c>0$. The lemma is proved.

Our next lemma provides a bootstrap version of Lemma 4.3.

Lemma 4.4. Suppose that the conditions of Lemma 4.2 hold true. Then

$$
\begin{aligned}
& \int_{\xi_{\alpha M: M}^{*}}^{\xi_{\alpha N: N}}\left(G(x)-G\left(\xi_{\alpha N: N}\right)\right) d F_{M}^{*}(x) \\
& \quad=-\frac{1}{2}\left[F_{M}^{*}\left(\xi_{\alpha N: N}\right)-F_{N}\left(\xi_{\alpha N: N}\right)\right]^{2} g\left(\xi_{\alpha N: N}\right) / f\left(\xi_{\alpha N: N}\right)+R_{M}^{*},
\end{aligned}
$$

where $P^{*}\left(\left|R_{M}^{*}\right|>M^{-1-p}\right)=o\left(M^{-c}\right)$ for every $p$ satisfying (2.20), with probability one, as $\min (N, M) \rightarrow \infty$.

The proof of Lemma 4.4 is relegated to Section 7.

Bias approximations. Next, we state and prove our lemmas on the asymptotic approximation for the bias of $T_{N}$ and $S_{N}$ in estimating of $\mu(\alpha, \beta)$ and for the bias of their bootstrap counterpart. Take some fixed $\delta>0$ such that $\xi_{\alpha}-\delta$ and $\xi_{\beta}+\delta$ belong to the neighborhoods, where the density $f$ is positive and Hölder continuous. Define auxiliary r.v.'s $X_{i}^{\prime}=\left(\xi_{\alpha}-\delta\right) \vee\left(X_{i} \wedge\left(\xi_{\beta}+\delta\right)\right)$, let $F^{\prime}$ and $F_{N}^{\prime}$ denote the $d f$ and the empirical $d f$ of the r.v. $X_{i}^{\prime}$ respectively, and let $X_{i: N}^{\prime}$ be the corresponding order statistics. Define auxiliary trimmed means

$$
T_{N}^{\prime}=\frac{1}{\beta-\alpha} \int_{\alpha}^{\beta}\left(F_{N}^{\prime}\right)^{-1}(u) d u \quad \text { and } \quad S_{N}^{\prime}=\frac{1}{([\beta N]-[\alpha N])} \sum_{i=[\alpha N]+1}^{[\beta N]} X_{i: N}^{\prime} .
$$


Under our smoothness conditions

$$
\sup _{x \in R}\left|P\left(T_{N} \leq x\right)-P\left(T_{N}^{\prime} \leq x\right)\right|=O\left(e^{-c_{\delta} N}\right),
$$

for some constant $c_{\delta}>0$ independent of $N$ (the same fact is valid for $S_{N}$ instead of $T_{N}$, cf. [11, 10]). Therefore, in absence of any moment assumptions on the distribution $F$, we can replace with impunity $T_{N}$ by $T_{N}^{\prime}$ (which has finite moments of arbitrary order) when proving Theorems 2.1 and 2.3. That is why we approximate the bias of the auxiliary r.v. $T_{N}^{\prime}$, and since the bootstrapped trimmed mean $T_{N, M}^{*}$ has finite moments of arbitrary order (in the bootstrap world) we can approximate its own bias. A similar comment applies to $S_{N}$.

Define

$$
\begin{array}{ll}
B_{N}=\sqrt{N}(\beta-\alpha)\left(E T_{N}^{\prime}-\mu(\alpha, \beta)\right), & B_{N, M}=\sqrt{M}(\beta-\alpha)\left(E^{*} T_{N, M}^{*}-T_{N}\right), \\
D_{N}=\sqrt{N}(\beta-\alpha)\left(E S_{N}^{\prime}-\mu(\alpha, \beta)\right), & D_{N, M}=\sqrt{M}(\beta-\alpha)\left(E^{*} S_{N, M}^{*}-T_{N}\right) .
\end{array}
$$

Lemma 4.5. Suppose that condition (C.1) is satisfied. Then for every $p<\min (a / 2,1 / 4)$

$$
\begin{aligned}
& B_{N}-b_{N}=o\left(N^{-1 / 2-p}\right), \\
& D_{N}-d_{N}=o\left(N^{-1 / 2-p}\right),
\end{aligned}
$$

with $b_{N}$ as in (2.13) and $d_{N}$ as in (2.15), as $N \rightarrow \infty$.

Moreover, if conditions $\left(C .1^{\prime}\right)$ and (C.2) hold, then for every p satisfying (2.20)

$$
\begin{array}{ll}
B_{N, M}-b_{N, M}=o\left(M^{-1 / 2-p}\right) & \text { a.s., } \\
D_{N, M}-d_{N, M}=o\left(M^{-1 / 2-p}\right) & \text { a.s., }
\end{array}
$$

with $b_{N, M}$ as in (2.19) and $d_{N, M}$ as in (2.26), as $\min (N, M) \rightarrow \infty$.

Proof. To prove relation (4.17) we use here the method, which corresponds to our general approach based on Bahadur's type approximation (cf. Lemma A.1 in [11], where we employed conditioning arguments to get a related result). Write

$$
\begin{aligned}
B_{N} & =\sqrt{N}(\beta-\alpha)\left(E T_{N}^{\prime}-\mu(\alpha, \beta)\right)=\sqrt{N} E\left(\int_{\alpha}^{\beta}\left(F_{N}^{\prime}\right)^{-1}(u) d u-\int_{\alpha}^{\beta} F^{-1}(u) d u\right) \\
& =\sqrt{N} E\left(\int_{\alpha}^{F_{N}^{\prime}\left(\xi_{\alpha}\right)}\left(F_{N}^{\prime}\right)^{-1}(u) d u+\int_{F_{N}^{\prime}\left(\xi_{\beta}\right)}^{\beta}\left(F_{N}^{\prime}\right)^{-1}(u) d u+\int_{\xi_{\alpha}}^{\xi_{\beta}} x d\left(F_{N}^{\prime}-F\right)(x)\right) .
\end{aligned}
$$

Since $F^{\prime}(x)=F(x)$ if $x \in\left(\xi_{\alpha}-\delta, \xi_{\beta}+\delta\right)$, we find that $E\left(\int_{\xi_{\alpha}}^{\xi_{\beta}} x d\left(F_{N}^{\prime}-F\right)(x)\right)=0$. Moreover, as $E\left(\int_{\alpha}^{F_{N}^{\prime}\left(\xi_{\alpha}\right)} d u\right)=E\left(F_{N}^{\prime}\left(\xi_{\alpha}\right)-F\left(\xi_{\alpha}\right)\right)=0$ and $E\left(\int_{F_{N}^{\prime}\left(\xi_{\beta}\right)}^{\beta} d u\right)=0$, the latter reduces to

$$
\sqrt{N} E\left(\int_{\alpha}^{F_{N}^{\prime}\left(\xi_{\alpha}\right)}\left(\left(F_{N}^{\prime}\right)^{-1}(u)-\xi_{\alpha}\right) d u+\int_{F_{N}^{\prime}\left(\xi_{\beta}\right)}^{\beta}\left(\left(F_{N}^{\prime}\right)^{-1}(u)-\xi_{\beta}\right) d u\right) .
$$

Define $N_{\nu}^{\prime}=\sharp\left\{i: X_{i}^{\prime} \leq \xi_{\alpha}\right\}, \nu=\alpha, \beta$, then by Lemma 4.2 the latter expression is equal to

$$
\sqrt{N} E\left(-\frac{\left(N_{\alpha}^{\prime}-\alpha N\right)^{2}}{2 N^{2}} \frac{1}{f\left(\xi_{\alpha}\right)}+\frac{\left(N_{\beta}^{\prime}-\beta N\right)^{2}}{2 N^{2}} \frac{1}{f\left(\xi_{\beta}\right)}\right)+o\left(N^{-1 / 2-p}\right)=b_{N}+o\left(N^{-1 / 2-p}\right),
$$

for every $p<\min (a / 2,1 / 4)$. This implies (4.17).

It remains to note that (4.19) follows if we replace $N, F^{\prime}$, and $F_{N}^{\prime}$ by their bootstrap counterparts: $M, F_{N}$, and $F_{M}^{*}$ in the preceding argument, and use the bootstrap versions of Lemmas 4.1 and 4.3 (i.e., 
relations (4.8) and (4.15) instead of (4.1) and (4.9)). Note that (4.19) follows also from (5.6)-(5.7) (see below) and the proof of Lemma 5.1.

Next we prove (4.18) and its bootstrap counterpart (4.20). Define the r.v.

$$
\tilde{S}_{N}^{\prime}=\frac{1}{N} \sum_{i=[\alpha N]+1}^{[\beta N]} X_{i: N}^{\prime}
$$

and write

$$
D_{N}=N^{1 / 2}(\beta-\alpha)\left(E S_{N}^{\prime}-\mu(\alpha, \beta)\right)=D_{1}+D_{2}
$$

where

$$
D_{1}=N^{1 / 2}\left((\beta-\alpha) E S_{N}^{\prime}-E \tilde{S}_{N}^{\prime}\right) \quad \text { and } \quad D_{2}=N^{1 / 2}\left(E \tilde{S}_{N}^{\prime}-(\beta-\alpha) \mu(\alpha, \beta)\right) .
$$

First consider $D_{2}$. Let us note that

$$
\tilde{S}_{N}^{\prime}=\frac{\alpha N-[\alpha N]}{N} X_{[\alpha N]+1: N}^{\prime}+\int_{\alpha}^{\beta}\left(F_{N}^{\prime}\right)^{-1}(u) d u-\frac{\beta N-[\beta N]}{N} X_{[\beta N]+1: N}^{\prime} .
$$

Therefore, by Lemma 4.1 we obtain

$$
D_{2}=N^{1 / 2}\left(\frac{\alpha N-[\alpha N]}{N} \xi_{\alpha}-\frac{\beta N-[\beta N]}{N} \xi_{\beta}+E\left(\int_{\alpha}^{\beta}\left(F_{N}^{\prime}\right)^{-1}(u) d u-\int_{\alpha}^{\beta} F^{-1}(u) d u\right)+o\left(N^{-1-p}\right)\right)
$$

for every $p<\min (a / 2,1 / 4)$, and the latter equals to

$$
\frac{\alpha N-[\alpha N]}{N^{1 / 2}} \xi_{\alpha}-\frac{\beta N-[\beta N]}{N^{1 / 2}} \xi_{\beta}+b_{N}+o\left(N^{-1 / 2-p}\right)
$$

(cf. proof of (4.17)). For $D_{1}$ we have

$$
D_{1}=N^{1 / 2}\left(\frac{N(\beta-\alpha)}{[\beta N]-[\alpha N]}-1\right) E \tilde{S}_{N}^{\prime}=N^{1 / 2}\left(\frac{\beta N-[\beta N]-\alpha N+[\alpha N]}{[\beta N]-[\alpha N]} E \tilde{S}_{N}^{\prime}\right)
$$

and taking into account the previous computations for $D_{2}$, we find that the last quantity is equal to

$$
\left(\frac{\beta N-[\beta N]}{N^{1 / 2}}-\frac{\alpha N-[\alpha N]}{N^{1 / 2}}\right) \mu(\alpha, \beta)+O\left(N^{-3 / 2}\right) .
$$

Relations (4.21)-(4.23) together imply that

$$
D_{1}+D_{2}=b_{N}+b_{[\cdot], N}+o\left(N^{-1 / 2-p}\right)=d_{N}+o\left(N^{-1 / 2-p}\right)
$$

for every $p<\min (a / 2,1 / 4)$, and (4.18) follows.

Finally note that (4.20) follows if we replace $N, F^{\prime}$, and $F_{N}^{\prime}$ by their bootstrap counterparts $M, F_{N}$, and $F_{M}^{*}$ in the preceding arguments and use the bootstrap versions of Lemmas 4.1 and 4.3. The lemma is proved.

\section{PROOF OF THEOREM 2.2}

To prove relation (2.21) we will need the bootstrap version of Lemma 4.1 in [11], which enables us to approximate $T_{N, M}^{*}$ by a $U$-statistic of degree two in the bootstrap world.

Define $\mathbf{1}_{\nu}\left(X_{i}^{*}\right)=\mathbf{1}_{\left\{X_{i}^{*} \leq \xi_{\nu N N}\right\}}$, where $\xi_{\nu N: N}=F_{N}^{-1}(\nu), 0<\nu<1$, and $\mathbf{1}_{A}$ denotes the indicator of event $A$. Then we can write

$$
W_{i}^{*}=X_{i}^{*} \mathbf{1}_{\beta}\left(X_{i}^{*}\right)\left(1-\mathbf{1}_{\alpha}\left(X_{i}^{*}\right)\right)+\xi_{\alpha N: N} \mathbf{1}_{\alpha}\left(X_{i}^{*}\right)+\xi_{\beta N: N}\left(1-\mathbf{1}_{\beta}\left(X_{i}^{*}\right)\right)
$$


(cf. (2.4)). Consider a $U$-statistic of degree 2 defined by

$$
L_{M}^{*}+U_{M}^{*}=\sum_{i=1}^{M} L_{M, i}^{*}+\sum_{1 \leq i} \sum_{<j \leq M} U_{M,(i, j)}^{*},
$$

where

$$
\begin{aligned}
L_{M, i}^{*} & =\frac{1}{\sqrt{M}}\left(W_{i}^{*}-\mu_{W, N}\right) \\
& =\frac{1}{\sqrt{M}}\left[X_{i}^{*} \mathbf{1}_{\beta}\left(X_{i}^{*}\right)\left(1-\mathbf{1}_{\alpha}\left(X_{i}^{*}\right)\right)+\xi_{\alpha N: N} \mathbf{1}_{\alpha}\left(X_{i}^{*}\right)+\xi_{\beta N: N}\left(1-\mathbf{1}_{\beta}\left(X_{i}^{*}\right)\right)-\mu_{W, N}\right]
\end{aligned}
$$

with $\mu_{W, N}$ as in (2.7) and

$$
\begin{aligned}
U_{M,(i, j)}^{*}= & \frac{1}{M \sqrt{M}}\left[-\frac{1}{f\left(\xi_{\alpha N: N)}\right.}\left(\mathbf{1}_{\alpha}\left(X_{i}^{*}\right)-\alpha_{N}\right)\left(\mathbf{1}_{\alpha}\left(X_{j}^{*}\right)-\alpha_{N}\right)\right. \\
& \left.+\frac{1}{f\left(\xi_{\beta N: N)}\right)}\left(\mathbf{1}_{\beta}\left(X_{i}^{*}\right)-\beta_{N}\right)\left(\mathbf{1}_{\beta}\left(X_{j}^{*}\right)-\beta_{N}\right)\right]
\end{aligned}
$$

where $\alpha_{N}=F_{N}\left(\xi_{\alpha N: N}\right), \beta_{N}=F_{N}\left(\xi_{\beta N: N}\right)$. Note that $E^{*} L_{N, i}^{*}=0$ for all $i=1, \ldots, M ; E^{*} U_{N,(i, j)}^{*}=0$ and $E^{*}\left(L_{N, i}^{*} U_{N,(i, j)}^{*}\right)=0$ for all $i, j=1, \ldots, M(i \neq j)$. Similarly as in [11], we have that $\xi_{\alpha}-\delta \leq$ $\xi_{\alpha N: N}, \xi_{\beta N: N} \leq \xi_{\beta}+\delta$ for every $\delta>0$ with probability $1-O\left(-c_{\delta} N\right)$ for some $c_{\delta}$ independent of $N$. Let $O_{p}\left(M^{-s}\right)(s>0)$ denote a r.v. of order $O\left(M^{-s}\right)$ with probability $1-O\left(\exp \left(-c_{\delta} N\right)\right)$. Then we easily check that $\left(\sigma_{L_{M}^{*}+U_{M}^{*}}^{*}\right)^{2}=E^{*}\left(L_{M}^{*}+U_{M}^{*}\right)^{2}=\sigma_{W, N}^{2}+O_{p}\left(M^{-1}\right)$ with $\sigma_{W, N}^{2}$ as in (2.7), and also that

$$
E^{*}\left(L_{M}^{*}+U_{M}^{*}\right)^{3}=E\left(L_{M}^{*}\right)^{3}+3 E\left(\left(L_{M}^{*}\right)^{2} U_{M}^{*}\right)+O_{p}\left(M^{-3 / 2}\right)=\frac{1}{\sqrt{M}}\left(\gamma_{W, N}+3 \delta_{W, N}\right)+O_{p}\left(M^{-3 / 2}\right) .
$$

Therefore,

$$
E^{*}\left(\frac{L_{M}^{*}+U_{M}^{*}}{\sigma_{L_{M}^{*}+U_{M}^{*}}^{*}}\right)^{3}=\frac{\lambda_{1, N}+3 \lambda_{2, N}}{\sqrt{M}}+O_{p}\left(M^{-3 / 2}\right)
$$

with $\lambda_{1, N}$ and $\lambda_{2, N}$ as in (2.11).

Next note that (cf. (2.7))

$$
\mu_{W, N}=\frac{[\alpha N]}{N} \xi_{\alpha N: N}+\frac{1}{N} \sum_{i=[\alpha N]+1}^{[\beta N]} X_{i: N}+\frac{N-[\beta N]}{N} \xi_{\beta N: N}
$$

Define the auxiliary r.v. $\tilde{T}_{N, M}^{*}=\frac{1}{\sqrt{M}} \sum_{i=[\alpha M]+1}^{[\beta M]} X_{i: M}^{*}$, then $\sqrt{M}(\beta-\alpha)\left(T_{N, M}^{*}-T_{N}\right)$ is equal to

$$
\begin{aligned}
& -\frac{\alpha M-[\alpha M]}{\sqrt{M}} X_{[\alpha M]+1: M}^{*}+\tilde{T}_{N, M}^{*}+\frac{\beta M-[\beta M]}{\sqrt{M}} X_{[\beta M]+1: M}^{*} \\
& -\frac{\sqrt{M}}{N} \sum_{i=[\alpha N]+1}^{[\beta N]} X_{i: N}+\sqrt{M} \frac{\alpha N-[\alpha N]}{N} X_{[\alpha N]+1: N}-\sqrt{M} \frac{\beta N-[\beta N]}{N} X_{[\beta N]+1: N}
\end{aligned}
$$

(cf. (2.1), (2.3)). Using $\left(C .1^{\prime}\right)$ and (C.2) we can apply Lemma 4.2 to obtain that (5.3) reduces to

$$
\begin{aligned}
& -\frac{\alpha M-[\alpha M]}{\sqrt{M}} \xi_{\alpha N: N}+\tilde{T}_{N, M}^{*}+\frac{\beta M-[\beta M]}{\sqrt{M}} \xi_{\beta N: N} \\
& -\frac{\sqrt{M}}{N} \sum_{i=[\alpha N]+1}^{[\beta N]} X_{i: N}+\sqrt{M} \frac{\alpha N-[\alpha N]}{N} \xi_{\alpha N: N}-\sqrt{M} \frac{\beta N-[\beta N]}{N} \xi_{\beta N: N}+R_{M}^{*},
\end{aligned}
$$


where $R_{M}^{*}$ is of order $o\left(M^{-1 / 2-p}\right)$ for every $p<d /(2-d)$, with probability one. Indeed, replacing $X_{[\alpha M]+1: M}^{*}$ and $X_{[\beta M]+1: M}^{*}$ in (5.3) by $\xi_{\alpha N: N}$ and $\xi_{\beta N: N}$ will lead to an error of order

$$
O\left(\frac{1}{\sqrt{M}}(\log M / M)^{1 / 2}\right)=o(\log M / M)
$$

(cf. Lemma 4.2), and by conditions $\left(C .1^{\prime}\right)$ and (C.2), replacing $X_{[\alpha N]+1: N}$ and $X_{[\beta N]+1: N}$ by $\xi_{\alpha N: N}$ and $\xi_{\beta N: N}$ can give the error of order $O\left(\frac{\sqrt{M} \log N}{N^{2}}\right)=o\left(M^{-1 / 2-p}\right)$, for every $p<d /(2-d)$ (and hence for every $p$ satisfying $(2.20))$.

Comparing now (5.2) and (5.4), we see that (5.4) is equal to

$$
\tilde{T}_{N, M}^{*}+\frac{[\alpha M]}{\sqrt{M}} \xi_{\alpha N: N}+\frac{M-[\beta M]}{\sqrt{M}} \xi_{\beta N: N}-\sqrt{M} \mu_{W, N}+R_{M}^{*}
$$

with $R_{M}^{*}$ as in (5.4). Define the quantity:

$$
\tilde{\mu}_{M}(\alpha, \beta)=\sqrt{M} \mu_{W, N}-\frac{[\alpha M]}{\sqrt{M}} \xi_{\alpha N: N}-\frac{M-[\beta M]}{\sqrt{M}} \xi_{\beta N: N}+b_{N, M},
$$

where $b_{N, M}$ is the bias term defined in (2.19). Then (5.3)-(5.6) together imply

$$
\sqrt{M}(\beta-\alpha)\left(T_{N, M}^{*}-T_{N}\right)=\tilde{T}_{N, M}^{*}-\tilde{\mu}_{M}(\alpha, \beta)+b_{N, M}+R_{M}^{*} .
$$

The next lemma ensures that under the conditions of Theorem 2.2 the approximation of $T_{N, M}^{*}$ by a $U$-statistic of the form (5.1) has a remainder of order $o\left(M^{-1 / 2-p}\right)$ for every $p$ satisfying (2.20), with probability one.

Lemma 5.1. Suppose that the conditions of Theorem 2.2 hold true. Then

$$
P^{*}\left(\left|\tilde{T}_{N, M}^{*}-\tilde{\mu}_{M}\left(\alpha_{N}, \beta_{N}\right)-\left(L_{M}^{*}+U_{M}^{*}\right)\right|>M^{-1 / 2-p}\right)=o\left(M^{-c}\right),
$$

for every $p$ satisfying (2.20), with probability one, as $\min (N, M) \rightarrow \infty$.

Proof. Define the binomial r.v.'s (in the bootstrap world) $M_{\nu}^{*}=\sharp\left\{i: X_{i}^{*} \leq \xi_{\nu N: N}\right\}, \nu=\alpha, \beta$, and write

$$
\begin{aligned}
\tilde{T}_{N, M}^{*}-\frac{1}{\sqrt{M}} \sum_{i=1}^{M} W_{i}^{*}=\frac{1}{\sqrt{M}}\left\{\operatorname{sign}\left[M_{\alpha}^{*}-[\alpha M]\right] \sum_{i=([\alpha M]+1) \wedge\left(M_{\alpha}^{*}+1\right)}^{M_{\alpha}^{*} \vee[\alpha M]}\left(X_{i: M}^{*}-\xi_{\alpha N: N}\right)\right. \\
\left.-\operatorname{sign}\left[M_{\beta}^{*}-[\beta M]\right] \sum_{i=\left([\beta M] \wedge M_{\beta}^{*}\right)+1}^{[\beta M] \vee M_{\beta}^{*}}\left(X_{i: M}^{*}-\xi_{\beta N: N}\right)-[\alpha M] \xi_{\alpha N: N}-(M-[\beta M]) \xi_{\beta N: N}\right\}
\end{aligned}
$$

(cf. the proof of Lemma 4.1 in [11]). By Lemma 4.4 the latter quantity is equal to

$$
-\frac{\left(M_{\alpha}^{*}-\alpha_{N} M\right)^{2}}{2 M \sqrt{M}} \frac{1}{f\left(\xi_{\alpha N: N}\right)}+\frac{\left(M_{\beta}^{*}-\beta_{N} M\right)^{2}}{2 M \sqrt{M}} \frac{1}{f\left(\xi_{\beta N: N}\right)}-\frac{[\alpha M]}{\sqrt{M}} \xi_{\alpha N: N}-\frac{M-[\beta M]}{\sqrt{M}} \xi_{\beta N: N}+R_{M}^{*},
$$

where $\left|R_{M}^{*}\right|=o\left(M^{-1 / 2-p}\right)$ for every $p$ satisfying (2.20), with probability one. This implies that

$$
\tilde{T}_{N, M}^{*}-\tilde{\mu}_{M}(\alpha, \beta)-L_{M}^{*}-U_{M}^{*}=\frac{1}{2 \sqrt{M}} \bar{r}_{M}^{*}+R_{M}^{*},
$$

where

$$
\begin{aligned}
\bar{r}_{M}^{*}=\frac{1}{M} \sum_{i=1}^{M} & \left\{-\frac{1}{f\left(\xi_{\alpha N: N}\right)}\left[\left(\mathbf{1}_{\alpha}\left(X_{i}^{*}\right)-\alpha_{N}\right)^{2}-\alpha_{N}\left(1-\alpha_{N}\right)\right]\right. \\
& \left.+\frac{1}{f\left(\xi_{\beta N: N}\right)}\left[\left(\mathbf{1}_{\beta}\left(X_{i}^{*}\right)-\beta_{N}\right)^{2}-\beta_{N}\left(1-\beta_{N}\right)\right]\right\} .
\end{aligned}
$$


Note that $\bar{r}_{M}^{*}$ is the average of $M$ i.i.d. (conditionally) bounded (with probability $1-O\left(\exp \left(-c_{\delta} N\right)\right)$ for some $\left.c_{\delta}>0\right)$ and centered $\left(E^{*} \bar{r}_{M}^{*}=0\right)$ r.v.'s, and by Hoeffding's inequality (cf. [17])

$$
P^{*}\left(\left|\bar{r}_{M}^{*}\right|>A(\log M / M)^{1 / 2}\right)=O\left(M^{-c}\right)
$$

for every $c>0$ and some $A>0$, not depending on $M$, with probability one. Therefore, $\frac{1}{2} \bar{r}_{M} / \sqrt{M}$ on the r.h.s. of (5.9) is negligible for our purposes. This together with relation (5.9) implies (5.8). The lemma is proved.

Proof of Theorem 2.2. Using Lemma 5.1 and relation (5.7), we find that the $d f F_{T_{N, M}^{*}}^{*}(x)$ (cf. Section 2) is equal to

$$
P^{*}\left(\frac{L_{M}^{*}+U_{M}^{*}}{\sigma_{W, N}} \leq x-\frac{b_{N, M}}{\sigma_{W, N}}-\frac{R_{M}^{*}}{\sigma_{W, N}}\right)
$$

where $L_{M}^{*}+U_{M}^{*}$ is a $U$-statistic of degree two and $R_{M}^{*}$ is as in Lemma 4.1 (cf. (5.7), (5.9)). The probability that the kernel of $L_{M}^{*}+U_{M}^{*}$ is bounded, uniformly in $M$, is of order $1-\exp \left(-c_{\delta} N\right)$ with some $c_{\delta}>0$ (cf. [11]). This means that the moment assumptions of Theorem 1.2 of Bentkus et al. [3] are satisfied (cf. also [11]). To complete the proof we have to verify that Cramér's condition is also satisfied in the bootstrap world. Note that the bootstrap (conditional) canonical function is

$$
\begin{aligned}
g_{N, M}(x) & =E^{*}\left(L_{M}^{*}+U_{M}^{*} \mid X_{1}^{*}=x\right) \\
& =\frac{1}{\sqrt{M}}\left[x \mathbf{1}_{\beta}(x)\left(1-\mathbf{1}_{\alpha}(x)\right)+\xi_{\alpha N: N} \mathbf{1}_{\alpha}(x)+\xi_{\beta N: N}\left(1-\mathbf{1}_{\beta}(x)\right)-\mu_{W, N}\right],
\end{aligned}
$$

and therefore $g_{N, M}\left(X_{1}^{*}\right)=\frac{1}{\sqrt{M}}\left(W_{1}^{*}-\mu_{W, N}\right)$. It follows that we have to check that

$$
\limsup _{|t| \rightarrow \infty}\left|E^{*} \exp \left(i t W_{1}^{*}\right)\right| \leq l<1
$$

for some fixed $0<l<1$ independent of $N$. Obviously

$$
E^{*} \exp \left(i t W_{1}^{*}\right)=\frac{[\alpha N]}{N} e^{i t \xi_{\alpha N: N}}+\frac{1}{N} \sum_{i=[\alpha N]+1}^{[\beta N]} e^{i t X_{i: N}}+\frac{N-[\beta N]}{N} e^{i t \xi_{\beta N: N}} .
$$

The sum of the first and the third terms in (5.12) is in absolute value bounded by

$$
\left|\frac{[\alpha N]}{N} e^{i t \xi_{\alpha N: N}}+\frac{N-[\beta N]}{N} e^{i t \xi_{\beta N: N}}\right| \leq \alpha+1-\beta+O\left(N^{-1}\right) .
$$

Next we consider the second term on the r.h.s. of (5.12). Write

$$
\frac{1}{N} \sum_{i=[\alpha N]+1}^{[\beta N]} e^{i t X_{i: N}}=\frac{[\beta N]-[\alpha N]}{N} \frac{1}{[\beta N]-[\alpha N]} \sum_{i=[\alpha N]+1}^{[\beta N]} e^{i t X_{i: N}} .
$$

By conditioning on $X_{[\alpha N]: N}=u$ and $X_{[\beta N]+1: N}=v$ (we can take $u$ and $v$ from neighborhoods of radius $A(\log N / N)^{1 / 2}$ of the points $\xi_{\alpha}$ and $\xi_{\beta}$ respectively) the conditional distribution of

$$
\frac{1}{[\beta N]-[\alpha N]} \sum_{i=[\alpha N]+1}^{[\beta N]} e^{i t X_{i: N}}
$$

is the same as the distribution of an average of i.i.d. bounded r.v.'s having expectation $q_{u, v}(t)=E e^{i t X_{u, v}}$, where $X_{u, v}$ is a r.v. with $d f F_{u, v}(x)=(F(x)-F(u)) /(F(v)-F(u)), u<x<v\left(F_{u, v}(x)=0, x \leq u\right.$, and $\left.F_{u, v}(x)=1, x>v\right)$. Due to our smoothness condition the distribution $F_{u, v}$ has a nontrivial absolutely continuous component, and we can conclude that $\lim \sup _{|t| \rightarrow \infty}\left|q_{u, v}(t)\right|=q_{u, v}<1$. Moreover, by Hoeffding's [17] inequality 


$$
\begin{aligned}
P\left(\left|\frac{1}{[\beta N]-[\alpha N]} \sum_{i=[\alpha N]+1}^{[\beta N]} e^{i t X_{i: N}}-q_{u, v}(t)\right|>A(\log N / N)^{1 / 2} \mid X_{[\alpha N]: N}=u, X_{[\beta N]+1: N}\right. & =v) \\
& =O\left(N^{-c}\right)
\end{aligned}
$$

for every $c>0$ with some $A>0$ independent of $N$, $t$, and of $u, v$ (boundedness of summands, uniformly in $u, v$ and $t$ ). Now note that by our smoothness condition

$$
q_{u, v}(t)=\int_{-\infty}^{\infty} e^{i t x} d F_{u, v}(x)=\int_{-\infty}^{\infty} e^{i t x} d F_{\xi_{\alpha}, \xi_{\beta}}(x)+R_{N}=q_{\xi_{\alpha}, \xi_{\beta}}(t)+R_{N}
$$

and $\left|R_{N}\right| \leq C(\log N / N)^{1 / 2}$, where $C$ is a constant independent of $N, u, v$ and $t$. So, $\sup _{u, v} q_{u, v}<q<1$, where supremum is taken over all $u, v$ in the neighborhoods of the points $\xi_{\alpha}$ and $\xi_{\beta}$ respectively; $q$ is a constant independent of $N$. Thus, by (5.12) and (5.13) for all sufficiently large $N$ we obtain

$$
\limsup _{|t| \rightarrow \infty}\left|E^{*} \exp \left(i t W_{1}^{*}\right)\right| \leq \alpha+q(\beta-\alpha)+1-\beta=l<1 .
$$

This proves (5.11). Then we can apply Theorem 1.2 of Bentkus, Götze and van Zwet [3]. Define $\tilde{F}_{M}^{*}(x)=\Phi(x)-\phi(x) \frac{\lambda_{1, N}+3 \lambda_{2, N}}{6 \sqrt{M}}\left(x^{2}-1\right)$, with $\lambda_{1, N}$ and $\lambda_{2, N}$ as in (2.11). We can conclude that

$$
\sup _{x \in R}\left|P^{*}\left\{\frac{L_{M}^{*}+U_{M}^{*}}{\sigma_{W, N}} \leq x\right\}-\tilde{F}_{M}^{*}(x)\right|=O\left(M^{-1}\right)
$$

with probability one. For $R_{M}^{*}$ (cf. (5.10)) we have $\left|R_{M}^{*}\right|=o\left(M^{-1 / 2-p}\right)$, for every $p$ satisfying (2.20), with probability one. Therefore, since $\left(\tilde{F}_{M}^{*}\right)^{\prime}(x)$ and $x\left(\tilde{F}_{M}^{*}\right)^{\prime}(x)$ are bounded functions, we obtain that the $P^{*}$-probability (5.10) is equal to

$$
\tilde{F}_{M}^{*}(x)-\phi(x) \frac{b_{N, M}}{\sigma_{W, N}}+o\left(M^{-1 / 2-p}\right)=G_{N, M}(x)+o\left(M^{-1 / 2-p}\right)
$$

for every $p$ satisfying (2.20), with probability one. This proves (2.21) and Theorem 2.2.

\section{PROOF OF THEOREM 2.4}

Recall that $M_{\nu}^{*}=\sharp\left\{i: X_{i}^{*} \leq \xi_{\nu N: N}\right\}, \nu=\alpha, \beta$, and $\alpha_{N}=F_{N}\left(\xi_{\alpha N: N}\right), \beta_{N}=F_{N}\left(\xi_{\beta N: N}\right)$. To prove relation (2.25), we will need the following lemma, which is nothing but a bootstrap counterpart of Lemma 5.1 in [11].

Lemma 6.1. Suppose that the assumptions of Theorem 2.4 are satisfied. Then, for any $c>0$ and every $p$ satisfying $(2.20)$

$$
P^{*}\left(\left|\left(\sigma_{W, M}^{*}\right)^{2}-\sigma_{W, N}^{2}-V_{M}^{*}\right|>M^{-1 / 2-p}\right)=o\left(M^{-c}\right),
$$

with probability one, as $\min (N, M) \rightarrow \infty$, where

$$
\begin{aligned}
V_{M}^{*} & =V_{M, 1}^{*}+V_{M, 2}^{*}, \\
V_{M, 1}^{*} & =2 \alpha \frac{1}{f\left(\xi_{\alpha N: N}\right)} \frac{M_{\alpha}^{*}-\alpha_{N} M}{M}\left[\mu_{W, N}-\xi_{\alpha N: N}\right]+2(1-\beta) \frac{1}{f\left(\xi_{\beta N: N}\right)} \frac{M_{\beta}^{*}-\beta_{N} M}{M}\left[\mu_{W, N}-\xi_{\beta N: N}\right], \\
V_{M, 2}^{*} & =\frac{1}{M} \sum_{i=1}^{M}\left[\left(W_{i}^{*}-\mu_{W, N}\right)^{2}-\sigma_{W, N}^{2}\right] .
\end{aligned}
$$

Moreover,

$$
E^{*}\left(V_{M}^{*}\right)=0 ; \quad E^{*}\left(V_{M}^{*}\right)^{2}=O_{p}\left(M^{-1}\right) \quad \text { as } \quad M \rightarrow \infty .
$$


As in Section 5, $O_{p}\left(M^{-1}\right)$ is our shorthand notation for a term of order $M^{-1}$ on a set of $P$-probability $1-O\left(e^{-c_{\delta} N}\right)$ for some $c_{\delta}>0$.

Proof. Similarly to the proof of Lemma 5.1 in [11], we define the auxiliary quantity

$$
\left(S_{W}^{*}\right)^{2}=\frac{1}{M} \sum_{i=1}^{M}\left(W_{i}^{*}\right)^{2}-\left(\frac{1}{M} \sum_{i=1}^{M} W_{i}^{*}\right)^{2}
$$

First we establish that

$$
\left(\sigma_{W, M}^{*}\right)^{2}=\left(S_{W}^{*}\right)^{2}+V_{M, 1}^{*}+R_{M, 1}^{*}
$$

Here and elsewhere $R_{M, 1}^{*}, R_{M, 1}^{*(r)}, r=1,2, \ldots$, denote remainder terms, which are of smaller order than $M^{-1 / 2-p}$ with $P^{*}$-probability $1-o\left(M^{-c}\right)$, for every $p$ satisfying $(2.20)$, with probability one. We have

$$
\begin{aligned}
\left(\sigma_{W, M}^{*}\right)^{2}-\left(S_{W}^{*}\right)^{2}=[ & \frac{[\alpha M]}{M}\left(\xi_{\alpha M: M}^{*}\right)^{2}+\frac{1}{M} \sum_{i=[\alpha M]+1}^{[\beta M]}\left(X_{i: M}^{*}\right)^{2}+\frac{M-[\beta M]}{M}\left(\xi_{\beta M: M}^{*}\right)^{2} \\
& \left.-\frac{M_{\alpha}^{*}}{M}\left(\xi_{\alpha N: N}\right)^{2}-\frac{1}{M} \sum_{i=M_{\alpha}^{*}+1}^{M_{\beta}^{*}}\left(X_{i: M}^{*}\right)^{2}-\frac{M-M_{\beta}^{*}}{M}\left(\xi_{\beta N: N}\right)^{2}\right] \\
& +\left[\left(\frac{1}{M} \sum_{i=1}^{M} W_{i}^{*}\right)^{2}-\left(\mu_{W, M}^{*}\right)^{2}\right] .
\end{aligned}
$$

Rewrite now the term in the first square brackets on the r.h.s. of (6.3) as

$$
\begin{aligned}
& \frac{\operatorname{sign}\left(M_{\alpha}^{*}-[\alpha M]\right)}{M} \sum_{i=\left([\alpha M] \wedge M_{\alpha}^{*}\right)+1}^{[\alpha M] \vee M_{\alpha}^{*}}\left(\left(X_{i: M}^{*}\right)^{2}-\left(\xi_{\alpha N: N}\right)^{2}\right) \\
& -\frac{\operatorname{sign}\left(M_{\beta}^{*}-[\beta M]\right)}{M} \sum_{i=\left([\beta M] \wedge M_{\beta}^{*}\right)+1}^{[\beta M] \vee M_{\beta}^{*}}\left(\left(X_{i: M}^{*}\right)^{2}-\left(\xi_{\beta N: N}\right)^{2}\right) \\
& +\frac{[\alpha M]}{M}\left(\left(\xi_{\alpha M: M}^{*}\right)^{2}-\left(\xi_{\alpha N: N}\right)^{2}\right)+\frac{M-[\beta M]}{M}\left(\left(\xi_{\beta M: M}^{*}\right)^{2}-\left(\xi_{\beta N: N}\right)^{2}\right) .
\end{aligned}
$$

By Lemmas 4.2 and 4.4 we find that the r.h.s. of (6.4) can be replaced by

$$
-2 \alpha \xi_{\alpha N: N} \frac{1}{f\left(\xi_{\alpha N: N}\right)} \frac{M_{\alpha}^{*}-\alpha_{N} M}{M}-2(1-\beta) \xi_{\beta N: N} \frac{1}{f\left(\xi_{\beta N: N}\right)} \frac{M_{\beta}^{*}-\beta_{N} M}{M}+R_{M, 1}^{*(1)},
$$

where the quadratic terms (cf. Lemma 4.4 above) are of negligible order and contribute to the remainder term $R_{M, 1}^{*(1)}$. Now consider the term in the second square brackets at the r.h.s. of (6.3). Arguing as before, we can rewrite this expression as

$$
\begin{aligned}
\left(\frac{2}{M}\right. & \left.\sum_{i=1}^{M} W_{i}^{*}-\alpha \frac{1}{f\left(\xi_{\alpha N: N}\right)} \frac{M_{\alpha}^{*}-\alpha_{N} M}{M}-(1-\beta) \frac{1}{f\left(\xi_{\beta N: N}\right)} \frac{M_{\beta}^{*}-\beta_{N} M}{M}+R_{M, 1}^{*(2)}\right) \\
& \times\left(\alpha \frac{1}{f\left(\xi_{\alpha N: N}\right)} \frac{M_{\alpha}^{*}-\alpha_{N} M}{M}+(1-\beta) \frac{1}{f\left(\xi_{\beta N: N)}\right)} \frac{M_{\beta}^{*}-\beta_{N} M}{M}+R_{M, 1}^{*(3)}\right) \\
& =\frac{2}{M}\left(\sum_{i=1}^{M} W_{i}^{*}\right)\left(\alpha \frac{1}{f\left(\xi_{\alpha N: N}\right)} \frac{M_{\alpha}^{*}-\alpha_{N} M}{M}+(1-\beta) \frac{1}{f\left(\xi_{\beta N: N}\right)} \frac{M_{\beta}^{*}-\beta_{N} M}{M}\right)+R_{M, 1}^{*(4)} .
\end{aligned}
$$


Relations (6.3)-(6.6) together imply that

$$
\left(\sigma_{W, M}^{*}\right)^{2}-\left(S_{W}^{*}\right)^{2}=V_{M, 1}^{*}+R_{M}^{*}+R_{M, 1}^{*(5)},
$$

where $R_{M}^{*}$ is equal to

$$
2\left[\alpha \frac{1}{f\left(\xi_{\alpha N: N}\right)} \frac{M_{\alpha}^{*}-\alpha_{N} M}{M}+(1-\beta) \frac{1}{f\left(\xi_{\beta N: N}\right)} \frac{M_{\beta}^{*}-\beta_{N} M}{M}\right] \frac{1}{M} \sum_{i=1}^{M}\left(W_{i}^{*}-\mu_{W, N}\right) .
$$

Note that $W_{i}^{*}, i=1, \ldots, M$, are uniformly bounded (with probability $1-\exp \left(-c_{\delta} N\right.$ ), for some $c_{\delta}>0$ ) i.i.d. r.v.'s. Therefore by Hoeffding's inequality and by using also Bernstein's inequality for the binomial r.v.'s $M_{\alpha}^{*}$ and $M_{\beta}^{*}$, we obtain that $\left|R_{M}^{*}\right|$ is of negligible order of magnitude for our purposes (cf. [11]).

Next we show that

$$
\left(S_{W}^{*}\right)^{2}=\left(\sigma_{W, N}\right)^{2}+V_{M, 2}^{*}+R_{M, 2}^{*},
$$

where $\left|R_{M, 2}^{*}\right|=o\left(M^{-1+\varepsilon}\right)$ with $P^{*}$-probability $1-o\left(M^{-c}\right)$, for every $\varepsilon, c>0$, a.s. $P$. We find that

$$
\left(S_{W}^{*}\right)^{2}-\left(\sigma_{W, N}\right)^{2}-V_{M, 2}^{*}=-\left(\bar{W}^{*}-\mu_{W, N}\right)^{2}=R_{M, 2}^{*} .
$$

An application of Hoeffding's inequality to the bounded (a.s. $[P])$ i.i.d. r.v.'s $W_{i}^{*}$ proves (6.7). Relations (6.2) and (6.7) together imply (6.1). The lemma is proved.

We are now in a position to prove Theorem 2.4. To establish the bootstrap version of the EE for a Studentized trimmed mean (i.e., relation (2.25)) we use the same argument as in the proof of Theorem 2.2 of [11], and employ Lemma 6.1 (i.e., the bootstrap version of Lemma 5.1 in [11]). The verification of Cramér's condition is the same as before (cf. the proof of Theorem 2.2). Theorem 2.4 is proved.

\section{PROOF OF LEMMAS 4.2 AND 4.4}

In this section we state and prove two lemmas, which will imply Lemmas 4.2 and 4.4 in Section 4. Throughout this section we suppose that the following condition holds true:

$\left(C .2^{\prime}\right) \quad \log N / N=O\left((\log M / M)^{1 / 2}\right) \quad$ as $\min (N, M) \rightarrow \infty$.

Note that when $(C .2)$ (cf. Section 2 ) holds true then $\left(C .2^{\prime}\right)$ is also automatically satisfied. In other words, assumption $\left(C .2^{\prime}\right)$ is a weaker requirement than $(C .2)$, but sufficient for our purposes here, i.e., for establishing Bahadur type results in the bootstrap world (cf. Lemmas 4.2 and 4.4). We will write $(g / f)(x)$ to denote the ratio $g(x) / f(x)$, set $M_{\alpha}^{*}=\sharp\left\{i: X_{i}^{*} \leq \xi_{\alpha N: N}\right\}$, and recall that $\alpha_{N}=F_{N}\left(\xi_{\alpha N: N}\right)$.

Lemma 7.1. Suppose that $f=F^{\prime}$ exists in a neighborhood of $\xi_{\alpha}$ and $f\left(\xi_{\alpha}\right)>0$. In addition, assume that the functions $f$ and $g$ satisfy the uniform Hölder condition of order $a>0$ in $a$ neighborhood of $\xi_{\alpha}$ and that condition $\left(C .2^{\prime}\right)$ holds true. Then

$$
G\left(\xi_{\alpha M: M}^{*}\right)-G\left(\xi_{\alpha N: N}\right)=-\left[F_{M}^{*}\left(\xi_{\alpha N: N}\right)-F_{N}\left(\xi_{\alpha N: N}\right)\right](g / f)\left(\xi_{\alpha N: N}\right)+R_{M}^{*},
$$

where

$$
\begin{array}{r}
P\left(P^{*}\left(\left|R_{M}^{*}\right|>A \max \left((\log M / M)^{(a+1) / 2},(\log M / M)^{3 / 4},(\log N / N)^{1 / 2}(\log M / M)^{1 / 4}\right)>M^{-c}\right)\right) \\
=O\left(N^{-c}\right)
\end{array}
$$

with some $A>0$ independent of $N$ and $M$, for every $c>0$, as $\min (N, M) \rightarrow \infty$.

This lemma implies Lemma 4.2 , because $(\log M / M)^{(a+1) / 2}=o\left(M^{-1 / 2-p}\right)$ for every $p<a / 2$, $(\log M / M)^{3 / 4}=o\left(M^{-1 / 2-p}\right)$ for every $p<1 / 4$ and by an application of $(C .2)$,

$$
(\log N / N)^{1 / 2}(\log M / M)^{1 / 4}=o\left(M^{-1 / 2-p}\right) \quad \text { for every } \quad p<d /(4(2-d)) .
$$

We can conclude that the maximum of these quantities is of order $o\left(M^{-1 / 2-p}\right)$ for every $p$ satisfying (2.20), and Lemma 4.2 follows. 
Proof. First we note (cf. (7.1))

$$
R_{M}^{*}=\frac{M_{\alpha}^{*}-\alpha_{N} M}{M}(g / f)\left(\xi_{\alpha N: N}\right)+G\left(\xi_{\alpha M: M}^{*}\right)-G\left(\xi_{\alpha N: N}\right)
$$

Similarly to the proof of Lemma 4.1 we first transform $R_{M}^{*}$ into a quantity involving uniform $(0,1)$ r.v.'s. Let $U_{1}, \ldots, U_{N}$ be a sample of $N$ independent, uniform $(0,1)$ distributed r.v.'s, let $F_{N, u}$ denote the corresponding empirical $d f$, and let $v_{\alpha N: N}=F_{N, u}^{-1}(\alpha)$ be the empirical $\alpha$-th quantile. Since the joint distribution of the $X_{i: N}, i=1, \ldots, N$, is the same as the joint distribution of $F^{-1}\left(U_{i: N}\right), i=1, \ldots, N$, we may as well take our bootstrap resample of size $M$ without replacement from $N$ independent uniform $(0,1)$ values $U_{1}, \ldots, U_{N}$. Note also that

$$
\alpha_{N}=F_{N}\left(\xi_{\alpha N: N}\right)=F_{N, u}\left(v_{\alpha N: N}\right)=N^{-1}([\alpha N]+\operatorname{sign}(\alpha N-[\alpha N])),
$$

where $\operatorname{sign}(0)=0$. Now it suffices to consider

$$
\frac{M_{\alpha, u}^{*}-\alpha_{N} M}{M}(g / f)\left(F^{-1}\left(v_{\alpha N: N}\right)\right)+G\left(F^{-1}\left(v_{\alpha M: M}^{*}\right)\right)-G\left(F^{-1}\left(v_{\alpha N: N}\right)\right),
$$

where $M_{\alpha, u}^{*}=\sharp\left\{i: U_{i}^{*} \leq v_{\alpha N: N}\right\}$ and $v_{\alpha M: M}^{*}$ is the $\alpha$-th quantile of the bootstrap sample $U_{1}^{*}, \ldots, U_{M}^{*}$. We can rewrite the second term of (7.2) as

$$
(g / f)\left(F^{-1}\left(v_{\alpha N: N}\right)\right)\left(v_{\alpha M: M}^{*}-v_{\alpha N: N}\right)+R_{M, 1}^{*}
$$

with $\left|R_{M, 1}^{*}\right| \leq C_{a}\left|v_{\alpha M: M}^{*}-v_{\alpha N: N}\right|^{a+1}$, where $C_{a}$ is the Hölder constant of the function $(g / f)\left(F^{-1}(u)\right)$ in a neighborhood of $\alpha$. Therefore we can rewrite (7.2) as

$$
(g / f)\left(F^{-1}\left(v_{\alpha N: N}\right)\right)\left[\frac{M_{\alpha, u}^{*}-\alpha_{N} M}{M}+v_{\alpha M: M}^{*}-v_{\alpha N: N}\right]+R_{M, 1}^{*} .
$$

Next we evaluate (7.3). First consider $R_{M, 1}^{*}$. We have

$$
P^{*}\left(\left|R_{M, 1}^{*}\right|>(A \log M / M)^{(1+a) / 2}\right) \leq P^{*}\left(\left|v_{\alpha M: M}^{*}-v_{\alpha N: N}\right|>C_{a}^{-1 /(a+1)}(A \log M / M)^{1 / 2}\right),
$$

where $A$ is some positive constant we will choose later. Set $A_{1}=A C_{a}^{-2 /(a+1)}$, then the r.h.s. of (7.4) is equal to

$$
P^{*}\left(v_{\alpha M: M}^{*}>v_{\alpha N: N}+\left(A_{1} \log M / M\right)^{1 / 2}\right)+P^{*}\left(v_{\alpha M: M}^{*}<v_{\alpha N: N}-\left(A_{1} \log M / M\right)^{1 / 2}\right) .
$$

We consider now only the first term in (7.5), since the second term can be treated in a completely similar fashion. Let $k_{\alpha}=\alpha_{N} N$ denote the index of the sample quantile $v_{\alpha N: N}$ among $U_{1: N} \leq \cdots \leq U_{N: N}$, and consider the binomial r.v. $S_{M}^{*}$ with parameters $\left(M, p^{*}\right)$ defined by

$$
S_{M}^{*}=\sharp\left\{i: U_{i}^{*} \leq U_{k_{\alpha}: N}+\left(A_{1} \log M / M\right)^{1 / 2}\right\}=\sum_{i=1}^{M} \mathbf{1}_{\left\{U_{i}^{*} \leq U_{k_{\alpha}: N}+\left(A_{1} \log M / M\right)^{1 / 2}\right\}},
$$

where $p^{*}=P^{*}\left(U_{i}^{*} \leq U_{k_{\alpha}: N}+\left(A_{1} \log M / M\right)^{1 / 2}\right)$. Note that $p^{*}$ is equal to

$$
N^{-1}\left(k_{\alpha}+\sharp\left\{i: U_{k_{\alpha}: N}<U_{i} \leq U_{k_{\alpha}: N}+\left(A_{1} \log M / M\right)^{1 / 2}\right\}\right) .
$$

Then

$$
E^{*} S_{M}^{*}=\frac{M}{N}\left(k_{\alpha}+\sharp\left\{i: U_{k_{\alpha}: N}<U_{i} \leq U_{k_{\alpha}: N}+\left(A_{1} \log M / M\right)^{1 / 2}\right\}\right) .
$$

Let $k_{\alpha, M}=[\alpha M]+\operatorname{sign}(\alpha M-[\alpha M])$ be the index of $v_{\alpha M: M}^{*}$ among $U_{1: M}^{*} \leq \cdots \leq U_{M: M}^{*}$. Then, we can rewrite the first term of (7.5) as

$$
P^{*}\left(S_{M}^{*}<k_{\alpha, M}\right)=P^{*}\left(S_{M}^{*}-E^{*} S_{N}^{*}<k_{\alpha, M}-k_{\alpha} \frac{M}{N}-M_{\alpha}\right),
$$


where $M_{\alpha}=\frac{M}{N}\left(\sharp\left\{i: U_{k_{\alpha}: N}<U_{i} \leq U_{k_{\alpha}: N}+\left(A_{1} \log M / M\right)^{1 / 2}\right\}\right)$. Define

$$
N_{U_{i}}=\sharp\left\{i: U_{k_{\alpha}: N}<U_{i} \leq U_{k_{\alpha}: N}+\left(A_{1} \log M / M\right)^{1 / 2}\right\}
$$

and note that $N_{U_{i}}$ has the same distribution (in the 'real world') as $\sharp\left\{i: U_{i} \leq\left(A_{1} \log M / M\right)^{1 / 2}\right\}$, then Bernstein's inequality directly implies that

$$
\left|N_{U_{i}}-N\left(A_{1} \log M / M\right)^{1 / 2}\right| \leq N\left(B_{1} \log N / N\right)^{1 / 2}\left(A_{1} \log M / M\right)^{1 / 4}
$$

with probability $1-O\left(N^{-c}\right)$ for any $c>0$ and some $B_{1}>2 c\left(1+\sqrt{C_{0}}\right)$, where $C_{0}$ is a constant satisfying $\log N / N \leq C_{0}(\log M / M)^{1 / 2}$ (cf. condition $\left(C .2^{\prime}\right)$ ), for all sufficiently large $M$ and $N$. Since $(\log N / N)^{1 / 2} \leq C_{0}^{1 / 2}(\log M / M)^{1 / 4}$, the r.h.s. of (7.7) can be bounded by

$$
N\left(B_{1} C_{0} A_{1}^{1 / 2}\right)^{1 / 2}(\log M / M)^{1 / 2} .
$$

Therefore, with proper choice of the constants $A_{1}$ and $B_{1}$ such that $A_{2}=A_{1}-B_{1} C_{0} A_{1}^{1 / 2}>0$ we obtain that $M_{\alpha}>\left(A_{2} M \log M\right)^{1 / 2}$. Finally, note that

$$
\begin{aligned}
\left|k_{\alpha, M}-k_{\alpha} \frac{M}{N}\right| & =\left|[\alpha M]+\operatorname{sign}(\alpha M-[\alpha M])-\alpha_{N} N \frac{M}{N}\right| \\
& \leq\left|\alpha-\alpha_{N}\right| M+1 \leq \frac{M}{N}+1 \\
& \leq \frac{M}{\log N} C_{0}(\log M / M)^{1 / 2}+1=o(M \log M)^{1 / 2} .
\end{aligned}
$$

Then an application of Bernstein's inequality at the r.h.s. of (7.6) ensures the required bound

$$
P\left(P^{*}\left(\left|R_{M, 3}^{*}\right|>(A \log M / M)^{(1+a) / 2}\right)>M^{-c}\right) \leq B N^{-c},
$$

for every $c>0$, where $A, B>0$ are some constants independent of $M$ and $N$.

It remains (cf. (7.3)) to evaluate

$$
R_{M, 2}^{*}=\frac{M_{\alpha, u}^{*}-\alpha_{N} M}{M}+v_{\alpha M: M}^{*}-v_{\alpha N: N} .
$$

Since $M_{\alpha, u}^{*}$ has (conditionally on $\left.U_{1}, \ldots, U_{N}\right)$ a binomial distribution with parameters $\left(\alpha_{N}, M\right)$, Bernstein's inequality directly implies that $P^{*}\left(\left|M_{\alpha, u}^{*}-\alpha_{N} M\right|>\left(A_{B} M \log M\right)^{1 / 2}\right)=O\left(M^{-c}\right)$ for every $c>0$ (provided that $A_{B}>2 c \alpha_{N}\left(1-\alpha_{N}\right)$ ). Therefore, we can bound our remainder $R_{M, 2}^{*}$ on the event $E^{*}=\left\{\left|M_{\alpha, u}^{*}-\alpha_{N} M\right| \leq\left(A_{B} M \log M\right)^{1 / 2}\right\}$. Let, as before, $k_{\alpha, M}=[\alpha M]+\operatorname{sign}(\alpha M-[\alpha M])$. If $M_{\alpha, u}^{*} \geq k_{\alpha, M}$, then $v_{\alpha M: M}^{*} \leq v_{\alpha N: N}$ and if $M_{\alpha, u}^{*}<k_{\alpha, M}$, then $v_{\alpha M: M}^{*} \geq v_{\alpha N+1: N}$ (where $v_{\alpha N+1: N}$ is the next order statistic after $v_{\alpha N: N}$ among $U_{1: N}, \ldots, U_{N: N}$ ). Our proof is based on the fact (cf. Lemma A, Appendix) that conditionally on $M_{\alpha, u}^{*}$ on the event $E^{*}$ and provided that $k_{\alpha, M} \leq M_{\alpha, u}^{*}$ (otherwise we proceed in a similar way with respect to the interval $\left[v_{\alpha N+1: N}, 1\right)$ instead $\left.\left(0, v_{\alpha N: N}\right]\right)$ the bootstrap quantile $v_{\alpha M: M}^{*}$ is distributed as $k_{\alpha, M}$-th order statistic $W_{k_{\alpha, M}: M_{\alpha, u}^{*}}$ of the sample $W_{1}^{*}, \ldots, W_{M_{\alpha, u}^{*}}^{*}$ of size $M_{\alpha, u}^{*}$ from the discrete uniform distribution with the values $U_{1: N}, \ldots, U_{k_{\alpha}: N}$ (where $U_{k_{\alpha}: N}=v_{\alpha N: N}$ ) and the probability mass $1 / k_{\alpha}$ at each of them.

For the case $k_{\alpha, M} \leq M_{\alpha, u}^{*}$ we rewrite $R_{M, 2}^{*}$ (cf. (7.9)) as

$$
R_{M, 2}^{*}=v_{\alpha M: M}^{*}-U_{k_{\alpha}: N}+\frac{U_{k_{\alpha}: N}\left(M_{\alpha, u}^{*}-k_{\alpha, M}\right)}{M_{\alpha, u}^{*}}+R_{M, 3}^{*},
$$

where

$$
R_{M, 3}^{*}=\frac{M_{\alpha, u}^{*}-\alpha_{N} M}{M}-\frac{U_{k_{\alpha}: N}\left(M_{\alpha, u}^{*}-k_{\alpha, M}\right)}{M_{\alpha, u}^{*}} .
$$


Since $\left|\alpha-\alpha_{N}\right| \leq 1 / N$ and $\left|k_{\alpha, M}-\alpha M\right| \leq 1$, the latter is equal to

$$
\begin{aligned}
\left(M_{\alpha, u}^{*}-k_{\alpha, M}\right)\left[\frac{1}{M}-\frac{U_{k_{\alpha}: N}}{M_{\alpha, u}^{*}}\right]+O\left(\frac{1}{N}+\frac{1}{M}\right) \\
=\frac{\left(M_{\alpha, u}^{*}-k_{\alpha, M}\right)\left(M_{\alpha, u}^{*}-M\left(\alpha+\left(U_{k_{\alpha}: N}-\alpha\right)\right)\right)}{M M_{\alpha, u}^{*}}+O\left(\frac{1}{N}+\frac{1}{M}\right) \\
=\frac{\left(M_{\alpha, u}^{*}-k_{\alpha, M}\right)^{2}}{M M_{\alpha, u}^{*}}-\frac{\left(M_{\alpha, u}^{*}-k_{\alpha, M}\right)\left(U_{k_{\alpha}: N}-\alpha\right)}{M_{\alpha, u}^{*}}+O\left(\frac{1}{N}+\frac{1}{M}\right) .
\end{aligned}
$$

The difference $M_{\alpha, u}^{*}-k_{\alpha, M}$ can be bounded in absolute value by

$$
\begin{aligned}
\left|M_{\alpha, u}^{*}-k_{\alpha, M}\right| & \leq\left|M_{\alpha, u}^{*}-\alpha_{N} M\right|+\left|\alpha_{N} M-k_{\alpha, M}\right| \leq\left(A_{B} M \log M\right)^{1 / 2}+\left|\alpha-\alpha_{N}\right| M+1 \\
& \leq\left(A_{B} M \log M\right)^{1 / 2}+\frac{M}{N}+1 \leq\left(A_{B} M \log M\right)^{1 / 2}+o(M \log M)^{1 / 2}
\end{aligned}
$$

(cf. (7.8)). Thus, the first term at the r.h.s. of $(7.11)$ is of order $O(\log M / M)$ on the event $E^{*}$ and by Lemma 4.1 the second term is of order $O\left((\log M / M)^{1 / 2}(\log N / N)^{1 / 2}\right)$ with $P^{*}$-probability $1-O\left(M^{-c}\right)$ with $P$-probability $1-O\left(N^{-c}\right)$ for every $c>0$. Finally, by condition $\left(C .2^{\prime}\right)$ we obtain that the latter is of order $O\left((\log M / M)^{3 / 4}\right)$. Hence, $R_{M, 3}^{*}$ contributes to the remainder term.

In the case $M_{\alpha, u}^{*}<k_{\alpha, M}$ we proceed in a similar way with respect to the interval $\left[v_{\alpha N+1: N}, 1\right)$ : now conditionally on $M_{\alpha, u}^{*}$ the bootstrap quantile $v_{\alpha M: M}^{*}=U_{k_{\alpha, M}: M}^{*}$ is distributed as $\left(k_{\alpha, M}-M_{\alpha, u}^{*}\right)$-th order statistic of the sample $W_{1}^{*}, \ldots, W_{M-M_{\alpha, u}^{*}}^{*}$ of size $M-M_{\alpha, u}^{*}$ from the discrete uniform distribution with values $U_{k_{\alpha}+1: N}, \ldots, U_{N: N}$ and the probability mass $1 /\left(N-k_{\alpha}\right)$ at each of them. And similarly on the event $E^{*}$ and $M_{\alpha, u}^{*}<k_{\alpha, M}$ we write

$$
R_{M, 2}^{*}=v_{\alpha M: M}^{*}-U_{k_{\alpha}+1: N}-\frac{\left(1-U_{k_{\alpha}+1: N}\right)\left(k_{\alpha, M}-M_{\alpha, u}^{*}\right)}{M-M_{\alpha, u}^{*}}+R_{M, 4}^{*},
$$

where

$$
R_{M, 4}^{*}=\frac{M_{\alpha, u}^{*}-\alpha_{N} M}{M}+\frac{\left(1-U_{k_{\alpha}+1: N}\right)\left(k_{\alpha, M}-M_{\alpha, u}^{*}\right)}{M-M_{\alpha, u}^{*}},
$$

and, as before, $R_{M, 4}^{*}$ is of order $O\left((\log M / M)^{3 / 4}+1 / N\right)$, i.e., contributes to the remainder term. It should be noted also that the spacing $v_{\alpha N+1: N}-v_{\alpha N: N}=O(\log N / N)$, and by condition $\left(C .2^{\prime}\right)$ it has the order of our remainder (cf. (7.1)).

Consider $R_{M, 2}^{*}$ given by (7.10) in the case $k_{\alpha, M} \leq M_{\alpha, u}^{*}$ (the treatment for (7.13) is similar). Relation (7.10) implies

$$
R_{M, 2}^{*}=v_{\alpha M: M}^{*}-\frac{U_{k_{\alpha}: N}}{M_{\alpha, u}^{*}} k_{\alpha, M}+R_{M, 3}^{*} .
$$

We have found (cf. above) that $R_{M, 3}^{*}$ is of order $O\left((\log M / M)^{3 / 4}+1 / N\right)$. Now we show that the quantity $v_{\alpha M: M}^{*}-\frac{U_{k_{\alpha}: N}}{M_{\alpha, u}^{*}} k_{\alpha, M}$ is of order $v(N, M)=(\log M / M)^{1 / 4} \max \left((\log N / N)^{1 / 2},(\log M / M)^{1 / 2}\right)$. Write

$$
\begin{aligned}
& P^{*}\left(\left|v_{\alpha M: M}^{*}-\frac{U_{k_{\alpha}: N} k_{\alpha, M}}{M_{\alpha, u}^{*}}\right|>A_{3} v(N, M) \mid M_{\alpha, u}^{*}: k_{\alpha, M} \leq M_{\alpha, u}^{*}\right) \\
& \quad=P_{W}^{*}\left(W_{k_{\alpha, M}: M_{\alpha, u}^{*}}>U_{k_{\alpha}: N} \frac{k_{\alpha, M}}{M_{\alpha, u}^{*}}+A_{3} v(N, M)\right) \\
& \quad+P_{W}^{*}\left(W_{k_{\alpha, M}: M_{\alpha, u}^{*}}<U_{k_{\alpha}: N} \frac{k_{\alpha, M}}{M_{\alpha, u}^{*}}-A_{3} v(N, M)\right)
\end{aligned}
$$


where $P_{W}^{*}$ denotes the conditional distribution, and $A_{3}$ is a positive constant, which may take different values in the course of the proof. Consider the first term in the r.h.s. of (7.15); our treatment of the second one is similar and therefore omitted. First note that we can assume that

$$
U_{k_{\alpha}: N} \frac{k_{\alpha, M}}{M_{\alpha, u}^{*}}+\left(A_{3}+t\right) v(N, M)<U_{k_{\alpha}: N}
$$

for some $t>\alpha c^{2}\left(A_{B}^{1 / 4}\right)$, where $A_{B}$ is a constant from the definition of the event $E^{*}$ and $c$ is an arbitrary constant (cf. the statement of the lemma), otherwise we can take a new $A_{3}$ equal to $A_{3}+t$, then the first term in the r.h.s. of (7.15) will be equal to zero, therefore, the necessary bound is trivial.

Define the binomial r.v.

$$
S_{M_{\alpha, u}^{*}}^{*}=\sum_{i=1}^{M_{\alpha, u}^{*}} \mathbf{1}_{\left\{W_{i}^{*} \leq \frac{U_{k_{\alpha}: N^{k} k_{\alpha, M}}}{M_{\alpha, u}^{*}}+A_{3} v(N, M)\right\}}
$$

with parameters $\left(p_{\alpha}^{*}, M_{\alpha, u}^{*}\right)$, where

$$
p_{\alpha}^{*}=P_{W}^{*}\left(W_{i}^{*} \leq \frac{U_{k_{\alpha}: N} k_{\alpha, M}}{M_{\alpha, u}^{*}}+A_{3} v(N, M)\right) .
$$

Then the first term at the r.h.s. of (7.15) equals

$$
P_{W}^{*}\left(S_{M_{\alpha, u}^{*}}^{*}<k_{\alpha, M}\right)=P_{W}^{*}\left(S_{M_{\alpha, u}^{*}}^{*}-M_{\alpha, u}^{*} p_{\alpha}^{*}<k_{\alpha, M}-M_{\alpha, u}^{*} p_{\alpha}^{*}\right) .
$$

Note that

$$
\begin{aligned}
p_{\alpha}^{*} & =\frac{1}{k_{\alpha}} \sharp\left\{i: U_{i} \leq \frac{k_{\alpha, M}}{M_{\alpha, u}^{*}} U_{k_{\alpha}: N}+A_{3} v(N, M)\right\} \\
& =\frac{1}{k_{\alpha}}\left(k_{\alpha}-\sharp\left\{i: \frac{k_{\alpha, M}}{M_{\alpha, u}^{*}} U_{k_{\alpha}: N}+A_{3} v(N, M)<U_{i} \leq U_{k_{\alpha}: N}\right\}\right) .
\end{aligned}
$$

As our estimation is in probability, we can replace the latter quantity by $1-\frac{1}{k_{\alpha}} N_{U_{i}}$, where

$$
N_{U_{i}}=\sharp\left\{i: U_{i}<\frac{M_{\alpha, u}^{*}-k_{\alpha, M}}{M_{\alpha, u}^{*}} U_{k_{\alpha}: N}-A_{3} v(N, M)\right\} .
$$

Now we note that by Bernstein's inequality

$$
\begin{aligned}
\mid N_{U_{i}} & -N\left(\frac{M_{\alpha, u}^{*}-k_{\alpha, M}}{M_{\alpha, u}^{*}} U_{k_{\alpha}: N}-A_{3} v(N, M)\right) \mid \\
& \leq B_{1}(\log N)^{1 / 2} N^{1 / 2}\left(\frac{M_{\alpha, u}^{*}-k_{\alpha, M}}{M_{\alpha, u}^{*}} U_{k_{\alpha}: N}-A_{3} v(N, M)\right)^{1 / 2},
\end{aligned}
$$

with probability $1-O\left(N^{-c}\right)$, where $B_{1}$ is a constant depending only on $c\left(B_{1}^{1 / 2}>c\right)$. Due to (7.16) the quantity in the r.h.s. of $(7.19)$ is less than $B_{1} N(\log N / N)^{1 / 2}\left(\frac{M_{\alpha, u}^{*}-k_{\alpha, M}}{M_{\alpha, u}^{*}} U_{k_{\alpha}: N}\right)^{1 / 2}$, and on the event $E^{*}$ the latter is less than $B_{1} N(\log N / N)^{1 / 2}\left(A_{B}\right)^{1 / 4}(\log M / M)^{1 / 4}$. Thus, with proper choice of $A_{3}$ $\left(A_{3}>B_{1}\left(A_{B}\right)^{1 / 4}\right)$ we get (cf. $\left.(7.18)-(7.19)\right)$ :

$$
p_{\alpha}^{*} \geq 1-\frac{1}{\alpha} \frac{M_{\alpha, u}^{*}-k_{\alpha, M}}{M_{\alpha, u}^{*}} U_{k_{\alpha}: N}+A_{4} v(N, M)
$$

with some $A_{4}>0$ independent of $M$ and $N$ and such that the quantity in the r.h.s. of (7.20) is less than 1 (cf. (7.16)). Now we get

$$
E^{*} S_{M_{\alpha, u}^{*}}^{*}=M_{\alpha, u}^{*} p_{\alpha}^{*} \geq M_{\alpha, u}^{*}\left(1-\frac{1}{\alpha} \frac{M_{\alpha, u}^{*}-k_{\alpha, M}}{M_{\alpha, u}^{*}} U_{k_{\alpha}: N}+A_{4} v(N, M)\right) .
$$


Note that $U_{k_{\alpha}: N}=\alpha-\frac{N_{\alpha}-\alpha N}{N}+R_{N}$ with $R_{N}=O_{p}\left((\log N / N)^{3 / 4}\right)$ (cf. Lemma 4.1), where $O_{p}($. denotes a quantity having the indicated order with probability $1-O\left(N^{-c}\right)$, for every $c>0$. Therefore, we obtain the following bound:

$$
\begin{aligned}
E^{*} S_{M_{\alpha, u}^{*}}^{*} & \geq M_{\alpha, u}^{*}\left(1-\frac{1}{\alpha} \frac{M_{\alpha, u}^{*}-k_{\alpha, M}}{M_{\alpha, u}^{*}}\left(\alpha-\frac{N_{\alpha}-\alpha N}{N}+R_{N}\right)+A_{4} v(N, M)\right) \\
& =k_{\alpha, M}+M_{\alpha, u}^{*}\left(\frac{1}{\alpha} \frac{M_{\alpha, u}^{*}-k_{\alpha, M}}{M_{\alpha, u}^{*}}\left(\frac{N_{\alpha}-\alpha N}{N}+R_{N}\right)+A_{4} v(N, M)\right) .
\end{aligned}
$$

Since $\left|N_{\alpha}-\alpha N\right| / N \leq A_{5}(\log N / N)^{1 / 2}$ with probability $1-O\left(N^{-c}\right)$ and $v(N, M) \geq(\log M / M)^{3 / 4}$, we obtain (with proper choice of $A_{3}$ ) that

$$
E^{*} S_{M_{\alpha, u}^{*}}^{*} \geq k_{\alpha, M}+A_{6} v(N, M) M_{\alpha, u}^{*} \geq k_{\alpha, M}+A_{7} M^{1 / 4}(\log M)^{3 / 4},
$$

and by (7.17) the first term in the r.h.s. of $(7.15)$ is not greater than

$$
P^{*}\left(S_{M_{\alpha, u}^{*}}^{*}-E^{*} S_{M_{\alpha, u}^{*}}^{*}<-A_{7} M^{1 / 4}(\log M)^{3 / 4}\right)
$$

with some positive $A_{7}$ independent of $N$ and $M$. By Bernstein's inequality the latter is less than $\exp (-h)$, where

$$
h=\frac{A_{7}^{2} M^{1 / 2}(\log M)^{3 / 2}}{2\left[M_{\alpha, u}^{*} p_{\alpha}^{*}\left(1-p_{\alpha}^{*}\right)+(1 / 3) A_{7} M^{1 / 4}(\log M)^{3 / 4} \max \left(p_{\alpha}^{*}, 1-p_{\alpha}^{*}\right)\right]} .
$$

It remains to note that (7.20) implies that

$$
1-p_{\alpha}^{*} \leq \frac{1}{\alpha} \frac{M_{\alpha, u}^{*}-k_{\alpha, M}}{M_{\alpha, u}^{*}} U_{k_{\alpha}: N}=O(\log M / M)^{1 / 2},
$$

therefore in the denominator of the last expression we have a quantity of exact order $O\left(M^{1 / 2}(\log M)^{1 / 2}\right)$. Thus, relations (7.14)-(7.15), (7.17), (7.21) and the latter estimate imply $\left|R_{M, 2}^{*}\right|=O(v(N, M))$, with $P^{*}$-probability $1-O\left(M^{-c}\right)$ with $P$-probability $1-O\left(N^{-c}\right)$ for any $c>0$. This, together with estimates for $R_{M, 1}^{*}$ (cf. (7.4)-(7.7)), imply (7.1). The lemma is proved.

The following lemma implies Lemma 4.4 .

Lemma 7.2. Suppose that the conditions of Lemma 7.1 are satisfied. Then

$$
\int_{\xi_{\alpha M: M}^{*}}^{\xi_{\alpha N: N}}\left(G(x)-G\left(\xi_{\alpha N: N}\right)\right) d F_{M}^{*}(x)=-\frac{1}{2}\left[F_{M}^{*}\left(\xi_{\alpha N: N}\right)-F_{N}\left(\xi_{\alpha N: N}\right)\right]^{2}(g / f)\left(\xi_{\alpha N: N}\right)+R_{M}^{*},
$$

where

$$
\begin{array}{r}
P\left(P^{*}\left(\left|R_{M}^{*}\right|>A \max \left((\log M / M)^{1+a / 2},(\log M / M)^{1+1 / 4},(\log N / N)^{1 / 2}(\log M / M)^{3 / 4}\right)>M^{-c}\right)\right) \\
=O\left(N^{-c}\right)
\end{array}
$$

with some constant $A>0$ independent of $N$ and $M$, for every $c>0$, as $\min (N, M) \rightarrow \infty$.

Again we note that if condition $(C .2)$ is satisfied, then

$$
\max \left((\log M / M)^{1+a / 2},(\log M / M)^{1+1 / 4},(\log N / N)^{1 / 2}(\log M / M)^{3 / 4}\right)=o\left(M^{-1-p}\right)
$$

for every $p$ satisfying (2.20), and Lemma 4.4 follows by Lemma 7.2. 
Proof. We need to estimate (cf. (7.22))

$$
R_{M}^{*}=\frac{\left(M_{\alpha}^{*}-\alpha_{N} M\right)^{2}}{2 M^{2}}(g / f)\left(\xi_{\alpha N: N}\right)+\frac{1}{M} \sum_{i=k_{\alpha, M}}^{M_{\alpha}^{*}}\left(G\left(X_{i: M}^{*}\right)-G\left(\xi_{\alpha N: N}\right)\right) .
$$

An argument similar the one used in the proof of Lemma 7.1 guarantees that we may as well consider

$$
\frac{\left(M_{\alpha, u}^{*}-\alpha_{N} M\right)^{2}}{2 M^{2}}(g / f)\left(F^{-1}\left(v_{\alpha N: N}\right)\right)+\frac{1}{M} \sum_{i=k_{\alpha, M}}^{M_{\alpha, u}^{*}}\left(G\left(F^{-1}\left(U_{i: M}^{*}\right)\right)-G\left(F^{-1}\left(v_{\alpha N: N}\right)\right)\right) .
$$

Our smoothness assumptions allow us to rewrite the latter quantity as

$$
(g / f)\left(F^{-1}\left(v_{\alpha N: N}\right)\right)\left[\frac{\left(M_{\alpha, u}^{*}-\alpha_{N} M\right)^{2}}{2 M^{2}}+\frac{1}{M} \sum_{i=k_{\alpha, M}}^{M_{\alpha, u}^{*}}\left(U_{i: M}^{*}-v_{\alpha N: N}\right)\right]+R_{M, 1}^{*},
$$

where

$$
\left|R_{M, 1}^{*}\right| \leq \frac{C_{a}\left|M_{\alpha, u}^{*}-k_{\alpha, M}\right|}{M} \max \left(\left|v_{\alpha M: M}^{*}-v_{\alpha N: N}\right|^{1+a},\left|U_{M_{\alpha, u}^{*}: M}^{*}-v_{\alpha N: N}\right|^{1+a}\right),
$$

where $C_{a}$ is a constant from the Hölder condition on the function $(g / f)\left(F^{-1}(u)\right)$ in a neighborhood of $\alpha$. We have proved earlier (cf. the proof of Lemma 7.1) that $\left|M_{\alpha, u}^{*}-k_{\alpha, M}\right| \leq\left(A_{B} M \log M\right)^{1 / 2}$ and $\left|v_{\alpha M: M}^{*}-v_{\alpha N: N}\right| \leq\left(A_{1} \log M / M\right)^{1 / 2}$ with $P^{*}$-probability $1-O\left(M^{-c}\right)$ with $P$-probability $1-O\left(N^{-c}\right)$ for every $c>0$, with some positive $A_{B}, A_{1}$ independent of $M$ and $N$, as $\min (N, M) \rightarrow \infty$. It remains to estimate $\left|U_{M_{\alpha, u}^{*}: M}^{*}-v_{\alpha N: N}\right|$. Conditionally on $M_{\alpha, u}^{*}$ on the event

$$
E^{*}=\left\{\left|M_{\alpha, u}^{*}-\alpha_{N} M\right| \leq\left(A_{B} M \log M\right)^{1 / 2}\right\}
$$

we have

$$
\begin{aligned}
& P^{*}\left(v_{\alpha N: N}-U_{M_{\alpha, u}^{*}: M}^{*}>\left(A_{2} \log M / M\right)^{1 / 2} \mid M_{\alpha, u}^{*}\right) \\
& \quad=P_{W}^{*}\left(W_{1: M_{\alpha, u}^{*}}^{*}>U_{1: N}+\left(A_{2} \log M / M\right)^{1 / 2}\right) \\
& \quad=\left(1-\frac{N_{U_{i}}}{k_{\alpha}}\right)^{M_{\alpha, u}^{*}},
\end{aligned}
$$

where $N_{U_{i}}=\sharp\left\{i: U_{i} \leq U_{1: N}+\left(A_{2} \log M / M\right)^{1 / 2}\right\} \geq N_{U_{i}}^{\prime}=\sharp\left\{i: U_{i} \leq\left(A_{2} \log M / M\right)^{1 / 2}\right\}$. As before (cf. (7.19)), we find that $\left|N_{U_{i}}^{\prime}-N\left(A_{2} \log M / M\right)^{1 / 2}\right| \leq B_{1} N(\log N / N)^{1 / 2}\left(A_{2} \log M / M\right)^{1 / 4}$, with probability $1-O\left(N^{-c}\right)$, where $B_{1}$ is a positive constant independent of $N$ and $M$. Condition $\left(C .2^{\prime}\right)$ directly implies that the latter quantity can be bounded by $B_{1} C_{0}^{1 / 2} A_{2}^{1 / 4} N(\log M / M)^{1 / 2}$, where as before (cf. the proof of Lemma 7.1) $C_{0}$ denotes a constant from condition $\left(C .2^{\prime}\right)$. So, with a proper choice of $A_{2}$, $A_{2}>B_{1} C_{0}^{1 / 2} A_{2}^{1 / 4}$, we have $N_{U_{i}}^{\prime} \geq N\left(A_{3} \log M / M\right)^{1 / 2}$ with some positive $A_{3}$. This implies that

$$
\left(1-\frac{N_{U_{i}}}{k_{\alpha}}\right)^{M_{\alpha, u}^{*}} \leq\left(1-\frac{N_{U_{i}}^{\prime}}{k_{\alpha}}\right)^{M_{\alpha, u}^{*}}=o\left(\exp \left(-M^{1 / 2}\right)\right) .
$$

This, together with (7.24) implies

$$
P^{*}\left(\left|R_{M, 1}^{*}\right|>A(\log M / M)^{1+a / 2}\right)=O\left(M^{-c}\right),
$$

for every $c>0$ and some constant $A>0$ independent of $M$ and $N$, with probability $1-O\left(N^{-c}\right)$.

Finally, consider the expression in square brackets in (7.23). Fix an arbitrary $c>0$ and take $c_{1}=$ $c+1$. Similarly to estimating $R_{M, 4}^{*}$ in the proof of Lemma 7.1 fix $M_{\alpha, u}^{*}$ on the event $E^{*}$, where 
$P^{*}\left(E^{*}\right)=1-O\left(M^{-c_{1}}\right)$ with probability 1 . If $M_{\alpha, u}^{*} \geq k_{\alpha, M}$, we can write

$$
\begin{aligned}
\frac{1}{M} \sum_{i=k_{\alpha, M}}^{M_{\alpha, u}^{*}}\left(U_{i: M}^{*}-v_{\alpha N: N}\right) & =\frac{1}{M} \sum_{i=k_{\alpha, M}}^{M_{\alpha, u}^{*}}\left(U_{i: M}^{*}-v_{\alpha N: N}+\frac{M_{\alpha, u}^{*}-i}{M}\right)-\frac{1}{M} \sum_{i=k_{\alpha, M}}^{M_{\alpha, u}^{*}} \frac{M_{\alpha, u}^{*}-i}{M} \\
& =-\frac{\left(M_{\alpha, u}^{*}-\alpha_{N} M\right)^{2}}{2 M^{2}}+O_{p}\left(M^{-3 / 2}\right)+R_{M, 2}^{*}
\end{aligned}
$$

where $O_{p}\left(M^{-3 / 2}\right)$ denotes a quantity of order $O\left(M^{-3 / 2}\right)$ with $P^{*}$-probability $1-O\left(M^{-c_{1}}\right)$, and

$$
R_{M, 2}^{*}=\frac{1}{M} \sum_{i=k_{\alpha, M}}^{M_{\alpha, u}^{*}}\left(U_{i: M}^{*}-\left(v_{\alpha N: N}-\frac{M_{\alpha, u}^{*}-i}{M}\right)\right) .
$$

When $M_{\alpha, u}^{*}<k_{\alpha, M}$, we write

$$
\begin{aligned}
\frac{1}{M} \sum_{i=k_{\alpha, M}}^{M_{\alpha, u}^{*}}\left(U_{i: M}^{*}-v_{\alpha N: N}\right) & =-\frac{1}{M} \sum_{i=M_{\alpha, u}^{*}}^{k_{\alpha, M}}\left(U_{i: M}^{*}-v_{\alpha N: N}\right) \\
& =-\frac{\left(M_{\alpha, u}^{*}-\alpha_{N} M\right)^{2}}{2 M^{2}}+O_{p}\left(M^{-3 / 2}\right)+R_{M, 3}^{*},
\end{aligned}
$$

where

$$
R_{M, 3}^{*}=-\frac{1}{M} \sum_{i=M_{\alpha, u}^{*}}^{k_{\alpha, M}}\left(U_{i: M}^{*}-\left(v_{\alpha N: N}+\frac{i-M_{\alpha, u}^{*}}{M}\right)\right) .
$$

Relations (7.23), (7.27)-(7.29) imply that it remains to prove that

$$
R_{M, 2}^{*} \mathbf{1}_{\left\{M_{\alpha, u}^{*} \geq k_{\alpha, M}\right\}}+R_{M, 3}^{*} \mathbf{1}_{\left\{M_{\alpha, u}^{*}<k_{\alpha, M}\right\}}
$$

is of order $O\left(\max \left[(\log N / N)^{1 / 2},(\log M / M)^{1 / 2}\right](\log M / M)^{3 / 4}\right)$ on the event $E^{*}$. We estimate $R_{M, 2}^{*}$, for $R_{M, 3}^{*}$ the treatment is similar. Consider the $i$-th term of the sum in (7.28). Similarly as in the proof of Lemma 7.1 we find that $U_{i: M}^{*}-\left(v_{\alpha N: N}-\frac{M_{\alpha, u}^{*}-i}{M}\right)$ can be replaced by

$$
U_{i: M}^{*}-\left(U_{k_{\alpha}: N}-\frac{U_{k_{\alpha}: N}\left(M_{\alpha, u}^{*}-i\right)}{M_{\alpha, u}^{*}}\right)=U_{i: M}^{*}-\frac{U_{k_{\alpha}: N} \cdot i}{M_{\alpha, u}^{*}} .
$$

Let, as before, $v(N, M)=\max \left((\log N / N)^{1 / 2},(\log M / M)^{1 / 2}\right)(\log M / M)^{1 / 4}$. Then

$$
\begin{gathered}
P^{*}\left(\left|U_{i: M}^{*}-\frac{U_{k_{\alpha}: N} \cdot i}{M_{\alpha, u}^{*}}\right|>A_{4} v(N, M) \mid M_{\alpha, u}^{*}: k_{\alpha, M} \leq M_{\alpha, u}^{*}\right) \\
\quad=P_{W}^{*}\left(\left|W_{i: M_{\alpha, u}^{*}}^{*}-\frac{U_{k_{\alpha}: N} \cdot i}{M_{\alpha, u}^{*}}\right|>A_{4} v(N, M)\right)
\end{gathered}
$$

with $W_{i: M_{\alpha, u}^{*}}^{*}$ and $P_{W}^{*}$ as in (7.15). Now we can repeat the arguments which were used in the proof of Lemma 7.1 (cf. (7.15)-(7.21)) to find that the r.h.s. of (7.30) is of order $O\left(M^{-c_{1}}\right)$, uniformly in $k_{\alpha, M} \leq$ $i \leq M_{\alpha, u}^{*}$, with probability $1-O\left(N^{-c_{1}}\right)$, for some constant $A_{4}$ independent of $M$ and $N$ (cf. [11]). Since the remainder term (7.28) contains $M_{\alpha, u}^{*}-k_{\alpha, M}<\left(A_{B} M \log M\right)^{1 / 2}$ such summands, we obtain that

$$
P^{*}\left(\left|R_{M, 2}^{*}\right|>A_{5}(\log M / M)^{3 / 4} v(N, M)\right)=O\left(M^{-c}\right),
$$

where $A_{5}$ is some positive constant independent of $M$ and $N$ (cf. the proof of Lemma 3.2 in [11]). This together with the estimates for $R_{M, 1}^{*}$ implies (7.22). The lemma is proved. 


\section{APPENDIX}

Let, as before, $X_{1}, \ldots, X_{N}$ be i.i.d. r.v.'s with common $d f F$, and $N_{\alpha}=\sharp\left\{i: X_{i} \leq \xi_{\alpha}\right\}, 0<\alpha<1$. Our proofs of Lemma 3.2 in [11] and Lemmas 4.2 and 4.3 in this paper use the following fact: conditionally on $N_{\alpha}$ the order statistics $X_{1: N}, \ldots, X_{N_{\alpha}: N}$ are distributed as the order statistics corresponding to a sample of $N_{\alpha}$ i.i.d. r.v.'s with distribution function $F(x) / \alpha, x \leq \xi_{\alpha}$. Though this fact is well known (cf. Theorem 12.4, [19], cf. also [11, 13]), we give a brief proof of it. The proof given here corrects an error in the argument given on p. 68 of [11]. Let $U_{1}, \ldots, U_{N}$ be independent r.v.'s uniformly distributed on $(0,1)$ and let $U_{1, N}, \ldots, U_{N, N}$ denote the corresponding order statistics. Put $N_{\alpha, u}=\sharp\left\{i: U_{i} \leq \alpha\right\}$.

Since $X_{i: N} \stackrel{\mathrm{d}}{=} F^{-1}\left(U_{i: N}\right)$ and $N_{\alpha} \stackrel{\mathrm{d}}{=} N_{\alpha, u}$, it is enough to prove the assertion for the uniform distribution.

Lemma A. Conditionally given $N_{\alpha, u}$, the order statistics $U_{1, N}, \ldots, U_{N_{\alpha, u}, N}$ are distributed as the order statistics corresponding to a sample of $N_{\alpha, u}$ independent $(0, \alpha)$-uniformly distributed r.v.'s.

Proof. (a) First consider the case $N_{\alpha, u}=N$. Take arbitrary $0<u_{1} \leq \cdots \leq u_{N}<\alpha$ and write

$$
\begin{aligned}
& P\left(U_{1: N} \leq u_{1}, \ldots, U_{N_{\alpha, u}: N} \leq u_{N} \mid N_{\alpha, u}=N\right) \\
& =\frac{P\left(U_{1: N} \leq u_{1}, \ldots, U_{N: N} \leq u_{N}\right)}{\alpha^{N}}=\frac{N !}{\alpha^{N}} \int_{0}^{u_{1}} \int_{x_{1}}^{u_{2}} \ldots \int_{x_{N-1}}^{u_{N}} d x_{1} d x_{2} \ldots d x_{N},
\end{aligned}
$$

and the latter is the d.f. of the order statistics corresponding to the sample of $N$ independent $(0, \alpha)$ uniformly distributed r.v.'s.

(b) Consider the case $N_{\alpha, u}=K<N$. The lemma follows now from the Markov property of order statistics and the validity of this fact for $N_{\alpha, u}=N$. More precisely, let

$$
F_{K, K+1}(u, v)=P\left(U_{K: N} \leq u, U_{K+1: N} \leq v\right), \quad u, v \in(0,1),
$$

be the joint d.f. of $U_{K: N}$ and $U_{K+1: N}$. Then for arbitrary $0<u_{1} \leq \cdots \leq u_{K}<\alpha$ we can write

$$
\begin{aligned}
& P\left(U_{1: N} \leq u_{1}, \ldots, U_{N_{\alpha, u}: N} \leq u_{K} \mid N_{\alpha, u}=K\right)=\left[P\left(N_{\alpha, u}=K\right)\right]^{-1} \\
& \quad \times \int_{\alpha}^{1} P\left(U_{1: N} \leq u_{1}, \ldots, U_{K: N} \leq u_{K} \mid U_{K: N} \leq \alpha, U_{K+1: N}=v>\alpha\right) d F_{K, K+1}(\alpha, v),
\end{aligned}
$$

and since conditionally given $U_{K+1: N}=v$ the order statistics $U_{1: N}, \ldots, U_{K: N}$ are distributed as order statistics corresponding to a sample of $K$ independent $(0, v)$-uniformly distributed r.v.'s, the latter expression is equal to

$$
\begin{array}{r}
{\left[P\left(N_{\alpha, u}=K\right)\right]^{-1} \frac{K !}{(\alpha / v)^{K}} \frac{1}{v^{K}} \int_{0}^{u_{1}} \int_{x_{1}}^{u_{2}} \ldots \int_{x_{K-1}}^{u_{K}} d x_{1} d x_{2} \ldots d x_{K} P\left(U_{K, N} \leq \alpha<U_{K+1: N}\right)} \\
=\frac{K !}{\alpha^{K}} \int_{0}^{u_{1}} \int_{x_{1}}^{u_{2}} \ldots \int_{x_{K-1}}^{u_{K}} d x_{1} d x_{2} \ldots d x_{K},
\end{array}
$$

which corresponds to the $(0, \alpha)$-uniform distribution. The lemma is proved.

\section{REFERENCES}

1. M. A. Arcones and E. Giné, "The bootstrap of the mean with arbitrary bootstrap sample size", Ann. Inst. H. Poincaré Probab. Statist. 25, 457-481 (1989).

2. R. R. Bahadur, "A note on quantiles in large samples", Ann. Math. Statist. 37, 577-580 (1966).

3. V. Bentkus, F. Götze, and W. R. van Zwet, "An Edgeworth expansion for symmetric statistics", Ann. Statist. 25, 851-896 (1997).

4. P. J. Bickel and D. A. Freedman, "Some asymptotic theory for the bootstrap", Ann. Statist. 9, 1196-1217 (1981). 
5. P. J. Bickel, F. Götze, and W. R. van Zwet, "The Edgeworth expansion for $U$-statistics of degree two", Ann. Statist. 14, 1463-1484 (1986).

6. P. J. Bickel, F. Götze, and W. R. van Zwet, "Resampling fewer than $n$ observations: gains, losses and remedies for losses", Statistica Sinica 7, 1-31 (1997).

7. P. J. Bickel and A. Sakov, "Extrapolation and the bootstrap", Sankhya Ser. A, Pt. 3, 64, 640-652 (2002).

8. B. Efron, "Bootstrap methods: another look at jackknife", Ann. Statist. 7, 1-26 (1979).

9. B. Efron and R. J. Tibshirani, An Introduction to the Bootstrap (Chapman and Hall, New York, 1993).

10. N. V. Gribkova, "On analogs of the Berry-Esseen inequality for truncated linear combinations of order statistics”, Theory Probab. Appl. 38, 142-149 (1993).

11. N. V. Gribkova and R. Helmers, "The empirical Edgeworth expansion for a Studentized trimmed mean", Math. Methods Statist. 15, 61-87 (2006).

12. P. Hall, The Bootstrap and Edgeworth Expansion (Springer, New York, 1992).

13. P. Hall and A. R. Padmanabhan, "On the bootstrap and the trimmed mean", J. Multivariate Anal. 41, 132$153(1992)$.

14. F. R. Hampel, E. M. Ronchetti, P. J. Rousseeuw, and W. A. Stahel, Robust Statistics. The Approach Based on Influence Functions (Wiley, New York, 1986).

15. R. Helmers, "On the Edgeworth expansion and the bootstrap approximation for a Studentized $U$-statistic", Ann. Statist. 19, 470-484 (1991).

16. R. Helmers, B.-Y. Jing, G. Qin, and W. Zhou, "Saddlepoint approximations to the trimmed mean", Bernoulli 10, 465-501 (2004).

17. W. Hoeffding, "Probability inequalities for sums of bounded random variables", J. Amer. Statist. Assoc. 58, 13-30 (1963).

18. P. J. Huber, Robust Statistics (Wiley, New York, 1981).

19. O. Kallenberg, Foundations of Modern Probability (Springer, New York, 2002).

20. J. C. Kiefer, "Deviations between the sample quantile process and the sample $d f$ ", in: Nonparametric Techniques in Statistical Inference, Ed. by M. Puri (Cambridge Univ. Press., London, 1970), pp. 299-319.

21. H. Putter and W. R. van Zwet, "Empirical Edgeworth expansions for symmetric statistics", Ann. Statist. 26, 1540-1569 (1998).

22. R.-D. Reiss, Approximate Distributions of Order Statistics with Applications to Nonparametric Statistics (Springer, New York, 1989).

23. A. Sakov, Using the $m$ out of $n$ Bootstrap in Hypothesis Testing, Ph.D. Thesis (University of California, Berkeley, 1998).

24. G. R. Shorack and J. A. Wellner, Empirical Processes with Application in Statistics (Wiley, New York, 1986).

25. K. Singh, "On the asymptotic accuracy of Efron's bootstrap", Ann. Statist. 9, 1187-1195 (1981).

26. S. M. Stigler, "The asymptotic distribution of the trimmed mean”, Ann. Statist. 1, 472-477 (1973). 